\title{
Measurement of the branching fraction of the $B^{0} \rightarrow D_{s}^{+} \pi^{-}$decay
}

\section{LHCb Collaboration ${ }^{\star}$}

CERN, 1211 Geneva 23, Switzerland

Received: 26 October 2020 / Accepted: 19 December 2020 / Published online: 12 April 2021

(C) CERN for the benefit of the LHCb collaboration 2021, corrected publication 2021

\begin{abstract}
A branching fraction measurement of the $B^{0} \rightarrow D_{s}^{+} \pi^{-}$decay is presented using proton-proton collision data collected with the $\mathrm{LHCb}$ experiment, corresponding to an integrated luminosity of $5.0 \mathrm{fb}^{-1}$. The branching fraction is found to be $\mathcal{B}\left(B^{0} \rightarrow D_{s}^{+} \pi^{-}\right)=(19.4 \pm$ $1.8 \pm 1.3 \pm 1.2) \times 10^{-6}$, where the first uncertainty is statistical, the second systematic and the third is due to the uncertainty on the $B^{0} \rightarrow D^{-} \pi^{+}, D_{s}^{+} \rightarrow K^{+} K^{-} \pi^{+}$and $D^{-} \rightarrow K^{+} \pi^{-} \pi^{-}$branching fractions. This is the most precise single measurement of this quantity to date. As this decay proceeds through a single amplitude involving a $b \rightarrow u$ charged-current transition, the result provides information on non-factorisable strong interaction effects and the magnitude of the Cabibbo-Kobayashi-Maskawa matrix element $V_{u b}$. Additionally, the collision energy dependence of the hadronisation-fraction ratio $f_{s} / f_{d}$ is measured through $\bar{B}_{s}^{0} \rightarrow D_{s}^{+} \pi^{-}$and $B^{0} \rightarrow D^{-} \pi^{+}$decays.
\end{abstract}

\section{Introduction}

To test the Cabibbo-Kobayashi-Maskawa (CKM) sector of the Standard Model (SM), it is crucial to perform accurate measurements of the quark-mixing matrix elements. Any discrepancy among the numerous measurements of CKM matrix elements could reveal effects from new particles or forces beyond the SM. The knowledge of the magnitude of the matrix element $V_{u b}$ governing the strength of $b \rightarrow u$ transitions is key in the consistency checks of the SM and its naturally motivated extensions $[1,2]$.

^e-mail: jordy.butter@cern.ch
The hadronic $B^{0} \rightarrow D_{s}^{+} \pi^{-}$decay ${ }^{1}$ proceeds in the SM through the $b \rightarrow u$ transition as shown in Fig. 1. Its branching fraction is proportional to $\left|V_{u b}\right|^{2}$,

$\mathcal{B}\left(B^{0} \rightarrow D_{s}^{+} \pi^{-}\right)=\Phi\left|V_{u b}\right|^{2}\left|V_{c s}\right|^{2}\left|F\left(B^{0} \rightarrow \pi^{-}\right)\right|^{2} f_{D_{s}^{+}}^{2}\left|a_{\mathrm{NF}}\right|^{2}$,

where $\Phi$ is a phase-space factor, $F\left(B^{0} \rightarrow \pi^{-}\right)$is a form factor, $f_{D_{s}^{+}}$is the $D_{s}^{+}$decay constant, $V_{c s}$ is the CKM matrix element representing $c \rightarrow s$ transitions, and $\left|a_{\mathrm{NF}}\right|$ encapsulates non-factorisable effects. The form factor and the decay constant can be obtained from light-cone sum rules $[3,4]$ and lattice QCD calculations [5,6], and since $\left|V_{c S}\right|$ is known to be close to unity, the $B^{0} \rightarrow D_{s}^{+} \pi^{-}$branching fraction can be used to probe the product $\left|V_{u b}\right|\left|a_{\mathrm{NF}}\right|$. The assumption of factorisation is expected to hold, i.e. $\left|a_{\mathrm{NF}}\right|$ is close to unity, for $B$ meson decays into a heavy and a light meson, where the $W$ emission of the decay corresponds to the light meson and the spectator quark forms part of the heavy meson. This is not the case for the $B^{0} \rightarrow D_{s}^{+} \pi^{-}$decay, as shown in Fig 1, and consequently $\left|a_{\mathrm{NF}}\right|$ may be significantly different from unity [7].

The measurement of the $B^{0} \rightarrow D_{S}^{+} \pi^{-}$branching fraction can also be used to estimate the ratio of the amplitudes of the Cabibbo-suppressed $B^{0} \rightarrow D^{+} \pi^{-}$and the Cabibbo-favoured $B^{0} \rightarrow D^{-} \pi^{+}$decays,

$r_{D \pi}=\left|\frac{A\left(B^{0} \rightarrow D^{+} \pi^{-}\right)}{A\left(B^{0} \rightarrow D^{-} \pi^{+}\right)}\right|$,

which is necessary for the measurement of charge-parity $(C P)$ asymmetries in $B^{0} \rightarrow D^{\mp} \pi^{ \pm}$decays [8-13]. Assuming $\mathrm{SU}(3)$ flavour symmetry, Eq. (2) can be written as $[14,15]$

$r_{D \pi}=\tan \theta_{c} \frac{f_{D^{+}}}{f_{D_{s}^{+}}} \sqrt{\frac{\mathcal{B}\left(B^{0} \rightarrow D_{s}^{+} \pi^{-}\right)}{\mathcal{B}\left(B^{0} \rightarrow D^{-} \pi^{+}\right)}}$,

where $\theta_{c}$ is the Cabibbo angle and $f_{D^{+}}$is the decay constant of the $D^{+}$meson. SU(3) symmetry breaking is caused by different non-factorisable effects in in $B^{0} \rightarrow D_{s}^{+} \pi^{-}$and $B^{0} \rightarrow D^{+} \pi^{-}$decays.

\footnotetext{
${ }^{1}$ Inclusion of charge-conjugate modes is implied unless explicitly stated.
} 


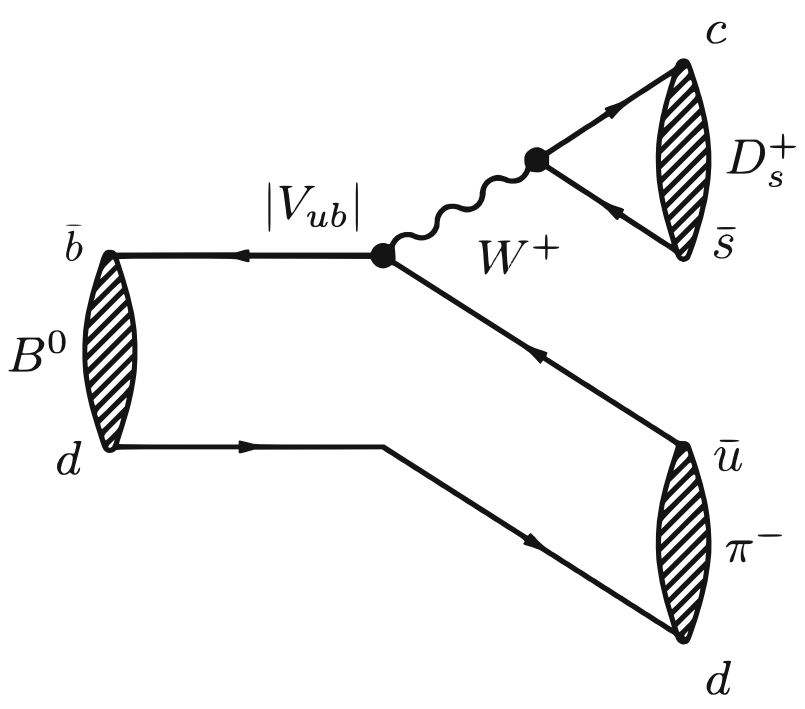

Fig. 1 Tree diagram of the $B^{0} \rightarrow D_{s}^{+} \pi^{-}$decay, in which a $B^{0}$ meson decays through the weak interaction to a $D_{s}^{+}$meson and a charged pion. This diagram represents the only (leading order) process contributing to this decay. Strong interaction between the $D_{s}^{+}$meson and the pion lead to a non-factorisable contribution to the decay amplitude

This article presents measurements of $\mathcal{B}\left(B^{0} \rightarrow D_{s}^{+} \pi^{-}\right)$ and $r_{D \pi}$ using proton-proton ( $p p$ ) collision data collected with the $\mathrm{LHCb}$ detector at centre-of-mass energies of 7,8 and $13 \mathrm{TeV}$ corresponding to an integrated luminosity of $5 \mathrm{fb}^{-1}$. The data samples recorded in the years 2011 and 2012 (2015 and 2016) at 7 and 8 (13) $\mathrm{TeV}$ will be referred to as Run 1 (Run 2). The $B^{0} \rightarrow D_{s}^{+} \pi^{-}$branching ratio is measured relative to the $B^{0} \rightarrow D^{-} \pi^{+}$normalisation channel, which is well measured and experimentally similar to the $B^{0} \rightarrow D_{s}^{+} \pi^{-}$ decay. The $B^{0} \rightarrow D_{s}^{+} \pi^{-}\left(B^{0} \rightarrow D^{-} \pi^{+}\right)$candidates are reconstructed via the $D_{s}^{+} \rightarrow K^{+} K^{-} \pi^{+}\left(D^{-} \rightarrow K^{+} \pi^{-} \pi^{-}\right)$decay. The branching fraction of the $B^{0} \rightarrow D_{s}^{+} \pi^{-}$decay is determined by

$$
\begin{aligned}
\mathcal{B}\left(B^{0} \rightarrow D_{s}^{+} \pi^{-}\right)= & \mathcal{B}\left(B^{0} \rightarrow D^{-} \pi^{+}\right) \frac{N_{B^{0} \rightarrow D_{s}^{+} \pi^{-}}}{N_{B^{0} \rightarrow D^{-} \pi^{+}}} \frac{\epsilon_{B^{0} \rightarrow D^{-} \pi^{+}}}{\epsilon_{B^{0} \rightarrow D_{s}^{+} \pi^{-}}} \\
& \times \frac{\mathcal{B}\left(D^{-} \rightarrow K^{+} \pi^{-} \pi^{-}\right)}{\mathcal{B}\left(D_{s}^{+} \rightarrow K^{+} K^{-} \pi^{+}\right)}
\end{aligned}
$$

where $N_{\mathrm{X}}$ denotes the selected candidate yield and $\epsilon_{\mathrm{X}}$ the related efficiency for the decay mode $X$. In this measurement, extended maximum-likelihood fits to unbinned invariant mass distributions are performed in order to obtain the yields, while the efficiencies are obtained from simulated events and using calibration data samples.

The relative production of $B_{s}^{0}$ and $B^{0}$ mesons, described by the ratio $f_{s} / f_{d}$ where $f_{s}$ and $f_{d}$ are the $B_{s}^{0}$ and $B^{0}$ hadronisation fractions, is shown to slightly depend on the $p p$ collision energy [16]. The efficiency-corrected yield ratio $\mathcal{R}$,

$\mathcal{R} \equiv \frac{N_{\bar{B}_{s}^{0} \rightarrow D_{s}^{+} \pi^{-}}}{N_{B^{0} \rightarrow D^{-} \pi^{+}}} \frac{\epsilon_{B^{0} \rightarrow D^{-} \pi^{+}}}{\epsilon_{\bar{B}_{s}^{0} \rightarrow D_{s}^{+} \pi^{-}}} \propto \frac{f_{s}}{f_{d}}$, is proportional to the relative production ratio and its dependence on the centre-of-mass energy is also reported here. This is measured using $\bar{B}_{s}^{0} \rightarrow D_{s}^{+} \pi^{-}$and $B^{0} \rightarrow D^{-} \pi^{+}$decays. Accurate knowledge of $f_{s} / f_{d}$ is a crucial input for every $B_{s}^{0}$ branching fraction measurement, e.g. $\mathcal{B}\left(B_{s}^{0} \rightarrow \mu^{+} \mu^{-}\right)$, since it dominates in most cases the systematic uncertainty [17]. Following the method described in Ref. [18], the value of $f_{s} / f_{d}$ can be calculated as

$\frac{f_{s}}{f_{d}}=0.982 \frac{\tau_{B_{d}}}{\tau_{B_{s}}} \frac{\mathcal{R}}{\mathcal{N}_{a} \mathcal{N}_{F} \mathcal{N}_{E}} \frac{\mathcal{B}\left(D^{-} \rightarrow K^{+} \pi^{-} \pi^{-}\right)}{\mathcal{B}\left(D_{s}^{+} \rightarrow K^{+} K^{-} \pi^{+}\right)}$,

where $\mathcal{R}$ is defined in Eq. (5), the numerical factor takes phase-space effects into account, $\mathcal{N}_{a}$ describes nonfactorisable $\mathrm{SU}(3)$ breaking effects, $\mathcal{N}_{F}$ is the ratio of the form factors, $\mathcal{N}_{E}$ takes into account the contribution of the $W$-exchange diagram in the $B^{0} \rightarrow D^{-} \pi^{+}$decay, and $\tau_{B_{d}}\left(\tau_{B_{s}}\right)$ is the $B^{0}\left(B_{s}^{0}\right)$ lifetime.

\section{Detector and simulation}

The LHCb detector $[19,20]$ is a single-arm forward spectrometer covering the pseudorapidity range $2<\eta<5$, designed for the study of particles containing $b$ or $c$ quarks. The detector includes a high-precision tracking system consisting of a silicon-strip vertex detector surrounding the $p p$ interaction region [21], a large-area silicon-strip detector located upstream of a dipole magnet with a bending power of about $4 \mathrm{Tm}$, and three stations of silicon-strip detectors and straw drift tubes [22,23] placed downstream of the magnet. The tracking system provides a measurement of the momentum, $p$, of charged particles with a relative uncertainty that varies from about $0.5 \%$ below $20 \mathrm{GeV} / c$ to $1.0 \%$ at $200 \mathrm{GeV} / c$. The minimum distance of a track to a primary vertex (PV), the impact parameter (IP), is measured with a resolution of $\left(15+29 / p_{\mathrm{T}}\right) \mu \mathrm{m}$, where $p_{\mathrm{T}}$ is the component of the momentum transverse to the beam, in $\mathrm{GeV} / c$. Different types of charged hadrons are distinguished using information from two ring-imaging Cherenkov (RICH) detectors [24]. Hadrons are identified by a calorimeter system consisting of scintillating-pad and preshower detectors, an electromagnetic and a hadronic calorimeter. Muons are identified by a system composed of alternating layers of iron and multiwire proportional chambers [25].

The online event selection is performed by a trigger [26], which consists of a hardware stage, based on information from the calorimeter and muon systems, followed by a software stage, which applies a full event reconstruction.

Simulation is required to calculate geometrical, reconstruction and selection efficiencies, and to determine shapes of invariant mass distributions. In the simulation, $p p$ collisions are generated using PYTHIA [27] with a specific LHCb configuration [28]. Decays of unstable particles are described 
by EVTGEN [29], in which final-state radiation is generated using Pнотоs [30]. The interaction of the generated particles with the detector, and its response, are implemented using the GEANT4 toolkit [31,32] as described in Ref. [33].

\section{Selection}

The $B^{0} \rightarrow D_{s}^{+} \pi^{-}\left(B^{0} \rightarrow D^{-} \pi^{+}\right)$decays are reconstructed by forming a $D_{S}^{+} \rightarrow K^{+} K^{-} \pi^{+}\left(D^{-} \rightarrow K^{+} \pi^{-} \pi^{-}\right)$candidate and combining it with an additional pion of opposite charge, referred to as the companion. The same reconstruction and selection procedure is applied to the $\bar{B}_{s}^{0} \rightarrow D_{s}^{+} \pi^{-}$decay. For the $B^{0} \rightarrow D_{s}^{+} \pi^{-}$decay, the invariant mass of the $K^{+} K^{-}$ pair is required to be within $20 \mathrm{MeV} / c^{2}$ of the $\phi(1020)$ mass to select only the $D_{s}^{+} \rightarrow \phi(1020) \pi^{+}$decays, which significantly improves the signal-to-background ratio compared to other decays with a $K^{+} K^{-} \pi^{+}$combination in the final state. Selecting $D_{s}^{+} \rightarrow \phi(1020) \pi^{+}$decays has an efficiency of about $40 \%$.

At the hardware trigger stage, events are required to have a muon with high $p_{\mathrm{T}}$ or a hadron, photon or electron with high transverse energy in the calorimeters. For hadrons, the transverse-energy threshold varied between 3 and $4 \mathrm{Ge} \mathrm{V}$ between 2011 and 2016. The software trigger requires a two-, three- or four-track secondary vertex with significant displacement from any primary $p p$ interaction vertex $(\mathrm{PV})$. At least one charged particle must have transverse momentum $p_{\mathrm{T}}>1.6 \mathrm{GeV} / c$ and be inconsistent with originating from a PV. A multivariate algorithm [34] is used for the identification of secondary vertices consistent with the decay of a $b$ hadron.

After the trigger selection, a preselection is applied to the reconstructed candidates to ensure good quality for the vertex of the $b$-hadron and $c$-hadron candidates comprising of tracks with large total and transverse momentum. Combinatorial background is suppressed using a gradient boosted decision tree (BDTG) algorithm [35,36], trained on Run 1 $\bar{B}_{s}^{0} \rightarrow D_{s}^{+} \pi^{-}$data. A set of 15 variables is used to train the BDTG classifier, the ones with highest importance in the training being the transverse momentum of the companion pion, the radial flight distance of the $\bar{B}_{s}^{0}$ and of the $D_{s}^{+}$candidates, the minimum transverse momentum of the $D_{s}^{+}$decay products and the minimum $\chi_{\mathrm{IP}}^{2}$ of the companion and the $\bar{B}_{s}^{0}$ candidates, where $\chi_{\mathrm{IP}}^{2}$ is defined as the difference in the vertex-fit $\chi^{2}$ of a given PV reconstructed with and without the particle under consideration. The correlation among the input variables has been studied and was found to be small. The BDTG classifier used in this measurement is described in Ref. [37].

To improve the $B^{0}$ and $\bar{B}_{s}^{0}$ invariant mass resolutions, the $D_{s}^{+}$and $D^{-}$invariant masses are constrained to their known values [38]. All $D_{s}^{+} \pi^{-}\left(D^{-} \pi^{+}\right)$candidates are required to have their invariant masses, $m\left(D_{s}^{+} \pi^{-}\right)\left(m\left(D^{-} \pi^{+}\right)\right)$, within the range 5150-5800 (5000-5800) MeV/c $c^{2}$ and the $K^{+} K^{-} \pi^{+}\left(K^{+} \pi^{-} \pi^{-}\right)$invariant mass within 1930-2065 (1830-1920) MeV/c $c^{2}$. The range of the $K^{+} K^{-} \pi^{+}$invariant mass includes a large upper sideband to model properly the combinatorial background shape, as described in Sect. 4.

To reduce the background due to misidentified final-state particles, particle identification (PID) information from the $\mathrm{RICH}$ detectors is used. The companion pion is required to pass a strict PID requirement to reduce the number of $\stackrel{\left(B_{s}^{0}\right.}{\rightarrow} D_{s}^{+} K^{-}\left(B^{0} \rightarrow D^{-} K^{+}\right)$decays where the kaon companion is misidentified as a pion. For $D_{s}^{+} \rightarrow \phi(1020) \pi^{+}$ candidates, loose PID requirements are applied to both kaons and the pion, which imply a signal efficiency of about $96 \%$. In the case of the pion, the PID requirement is used primarily to remove protons originating from the $\Lambda_{c}^{+} \rightarrow \phi p$ decay. Further PID requirements are applied to veto $\Lambda_{b}^{0} \rightarrow \Lambda_{c}^{+}\left(\rightarrow p K^{-} \pi^{+}\right) \pi^{-}$and $\bar{B}^{0} \rightarrow D^{+}\left(\rightarrow K^{-} \pi^{+} \pi^{+}\right) \pi^{-}$ and $\bar{\Lambda}_{b}^{0} \rightarrow \bar{\Lambda}_{c}^{-}\left(\rightarrow \bar{p} K^{+} \pi^{-}\right) \pi^{+}$and $B_{s}^{0} \rightarrow D_{s}^{-}\left(\rightarrow K^{-} K^{+} \pi^{-}\right) \pi^{+}$ events, which are misidentified as the final-state particles of $D_{s}^{+}\left(\rightarrow K^{+} K^{-} \pi^{+}\right) \pi^{-}$and $D^{-}\left(\rightarrow K^{+} \pi^{-} \pi^{-}\right) \pi^{+}$decays, respectively. These vetoes are applied if candidates are consistent with the above mentioned decays when a mass hypothesis is changed. The PID requirements result in $75 \%$ efficiency for $B^{0} \rightarrow D_{s}^{+} \pi^{-}$signal decays, which is dominated by the strict PID requirement on the companion pion, while the retention is about $9 \%$ for the $\stackrel{\overrightarrow{B_{s}^{0}}}{\rightarrow} \rightarrow D_{s}^{+} K^{-}$misidentified background contribution.

The event selection efficiencies are calculated from simulation with the exception of the efficiency of the PID requirements which is determined using calibration data samples.

\section{Signal and background parametrisation}

After the full event selection, unbinned maximum-likelihood fits are performed to obtain the yields of the signal $B^{0} \rightarrow D_{s}^{+} \pi^{-}$and the normalisation $B^{0} \rightarrow D^{-} \pi^{+}$candidates. A two-dimensional fit to the $D_{s}^{+} \pi^{-}$and the $K^{+} K^{-} \pi^{+}$ invariant mass distributions is performed to determine the $B^{0} \rightarrow D_{s}^{+} \pi^{-}$signal yield, while the yield of the normalisation channel is obtained from a fit to the $D^{-} \pi^{+}$invariant mass distribution. Due to the $D_{s}^{+}$mass constraint, the correlation between $m\left(D_{s}^{+} \pi^{-}\right)$and $m\left(K^{+} K^{-} \pi^{+}\right)$is found to be small, thus the two variables are factorised in the fit model [39]. The two-dimensional fit is performed in order to constrain the combinatorial background (see further in this Section for details).

The $B^{0} \rightarrow D_{s}^{+} \pi^{-}$decay is Cabibbo-suppressed and is therefore considerably less abundant than the Cabibbo- 
favoured $\bar{B}_{s}^{0} \rightarrow D_{s}^{+} \pi^{-}$decay, which produces the same finalstate particles. The $m\left(D_{s}^{+} \pi^{-}\right)$and $m\left(D^{-} \pi^{+}\right)$shapes for $\bar{B}_{s}^{0} \rightarrow D_{s}^{+} \pi^{-}$and $B^{0} \rightarrow D^{-} \pi^{+}$candidates, respectively, are described by the sum of a double-sided Hypatia function [40] and a Johnson $S_{U}$ function [41]. The left tail of the $\bar{B}_{s}^{0} \rightarrow D_{s}^{+} \pi^{-}$invariant mass distribution overlaps with the $B^{0} \rightarrow D_{s}^{+} \pi^{-}$signal peak and therefore special attention is given to the description of the lower mass range of the $\bar{B}_{s}^{0} \rightarrow D_{s}^{+} \pi^{-}$peak, shaped by the combination of detector resolution and radiative effects. The $B^{0} \rightarrow D_{s}^{+} \pi^{-}$signal is described with the same model as the $\bar{B}_{s}^{0} \rightarrow D_{s}^{+} \pi^{-}$decay, shifted by the known $B^{0}-B_{s}^{0}$ mass difference [38]. The left tail of this distribution is described by two parameters, $a_{1}$ and $n_{1}$, which are found to be correlated and therefore the parameter $n_{1}$ is fixed to the value obtained from simulation, whereas $a_{1}$ is obtained from simulated $\bar{B}_{s}^{0} \rightarrow D_{s}^{+} \pi^{-}$and $B^{0} \rightarrow D^{-} \pi^{+}$ events, as well as from $B^{0} \rightarrow D^{-} \pi^{+}$data. In the invariant mass fit to $B^{0} \rightarrow D^{-} \pi^{+}$candidates the common mean of the double-sided Hypatia and the Johnson $S_{U}$ functions, the widths and the left-tail parameter $a_{1}$ are left free in the fit, while this parameter is constrained in the $D_{s}^{+} \pi^{-}$invariant mass distribution, as the background does not allow to determine the shape of the radiative tail reliably. All other parameters are fixed from simulation. In the $K^{+} K^{-} \pi^{+}$invariant mass fit a sum of two Crystal Ball functions with a common mean is used. The common mean and a scale factor for the widths are left free, while the other shape parameters are fixed from simulation.

The combinatorial background in $B^{0} \rightarrow D_{s}^{+} \pi^{-}$candidates is split in two components, referred to as random- $D_{s}^{+}$and true- $D_{s}^{+}$. The random- $D_{s}^{+}$combinatorial background consists of random combinations of tracks that do not peak in the $K^{+} K^{-} \pi^{+}$invariant mass, while the true- $D_{s}^{+}$combinatorial background consists of events with a true $D_{s}^{+}$meson, combined with a random companion track. The upper mass range of the $K^{+} K^{-} \pi^{+}$candidate sample is used to account accurately for the random- $D_{s}^{+}$component, modelled with a single exponential distribution, while the true- $D_{s}^{+}$background is described by the signal shape. In the $D_{s}^{+} \pi^{-}$invariant mass fit, the random- $D_{s}^{+}$background is described by an exponential distribution and the true- $D_{s}^{+}$background is described by the sum of an exponential and a constant function. The exponential parameters are left free in both invariant mass fits.

The combinatorial background in the $m\left(D^{-} \pi^{+}\right)$fit of the normalisation channel is described by the sum of an exponential and a constant function, with the relative weight of the two functions and exponential parameter left free.
Decays where one or more final-state particles are not reconstructed are referred to as partially reconstructed backgrounds. In the $D_{s}^{+} \pi^{-}$and $D^{-} \pi^{+}$invariant mass fits these background contributions are described by an upward-open parabola or a parabola exhibiting a maximum, whose ranges are defined by the kinematic endpoints of the decay, which are convolved with Gaussian resolution functions, and which are known to describe decays involving a missing neutral pion or a missing photon, as defined in Ref. [42]. In the fit to the $K^{+} K^{-} \pi^{+}$invariant mass, the partially reconstructed background contributions are described by the signal mass shape.

The $m\left(D_{s}^{+} \pi^{-}\right)$fit requires two partially reconstructed background components from $\bar{B}_{s}^{0} \rightarrow D_{s}^{*+}\left(\rightarrow D_{s}^{+} \gamma / \pi^{0}\right) \pi^{-}$ and $\bar{B}_{s}^{0} \rightarrow D_{s}^{+} \rho^{-}\left(\rightarrow \pi^{-} \pi^{0}\right)$ decays. The fit model describing the $D^{-} \pi^{+}$invariant mass accounts analogously for two partially reconstructed background contributions: $B^{0} \rightarrow D^{*-}\left(\rightarrow D^{-} \pi^{0}\right) \pi^{+}$and $B^{0} \rightarrow D^{-} \rho^{+}\left(\rightarrow \pi^{+} \pi^{0}\right)$. In the case of the $\bar{B}_{s}^{0} \rightarrow D_{s}^{*+} \pi^{-}$background the previously mentioned upward-open parabola together with a parabola exhibiting a maximum is used to parameterise the components with $D_{s}^{*+} \rightarrow D_{s}^{+} \gamma$ and $D_{s}^{*+} \rightarrow D_{s}^{+} \pi^{0}$ decays, respectively. The $\bar{B}_{s}^{0} \rightarrow D_{s}^{+} \rho^{-}$background is described by the upward-open parabola, to take into account the missing neutral pion. The $B^{0} \rightarrow D^{*-} \pi^{+}$decay uses an upward-open parabola function and exhibits a double-peaked shape. Most parameters are obtained from simulated events and fixed, aside from the relevant invariant mass shifts and widths. For the $B^{0} \rightarrow D^{-} \rho^{+}$background a single upward-open parabola function is taken, with a floating width and a floating mass shift parameter that is shared with the $B^{0} \rightarrow D^{*-} \pi^{+}$contribution. The widths of the partially reconstructed background contributions in the $m\left(D_{s}^{+} \pi^{-}\right)$fits are fixed to the values obtained from $B^{0} \rightarrow D^{-} \pi^{+}$candidates in data, corrected for differences between the $m\left(D_{s}^{+} \pi^{-}\right)$and $m\left(D^{-} \pi^{+}\right)$distributions, as obtained from simulation.

The $B^{0} \rightarrow D^{-} \pi^{+}$candidate sample is contaminated by the $B_{s}^{0} \rightarrow D_{s}^{-} \pi^{+}, \bar{\Lambda}_{b}^{0} \rightarrow \bar{\Lambda}_{c}^{-} \pi^{+}$and $B^{0} \rightarrow D^{-} K^{+}$decays, resulting from the misidentification of one or two of the final-state particles. Analogously, the $\stackrel{\overrightarrow{B_{s}^{0}}}{\rightarrow} \rightarrow D_{s}^{+} K^{-}, \Lambda_{b}^{0} \rightarrow \Lambda_{c}^{+} \pi^{-}$and $\bar{B}^{0} \rightarrow D^{+} \pi^{-}$decays are misidentified background contributions of the $B^{0} \rightarrow D_{s}^{+} \pi^{-}$candidate sample. Their shapes are determined from simulation using a non-parametric kernel estimation method [43]. The yields of the misidentified background contributions are estimated by using known branching fractions [38] and efficiencies that are determined from simulated background decays. Each yield of a misidentified background in the fit model is constrained to be close to its estimated value and is allowed to vary within the corresponding uncertainty. 

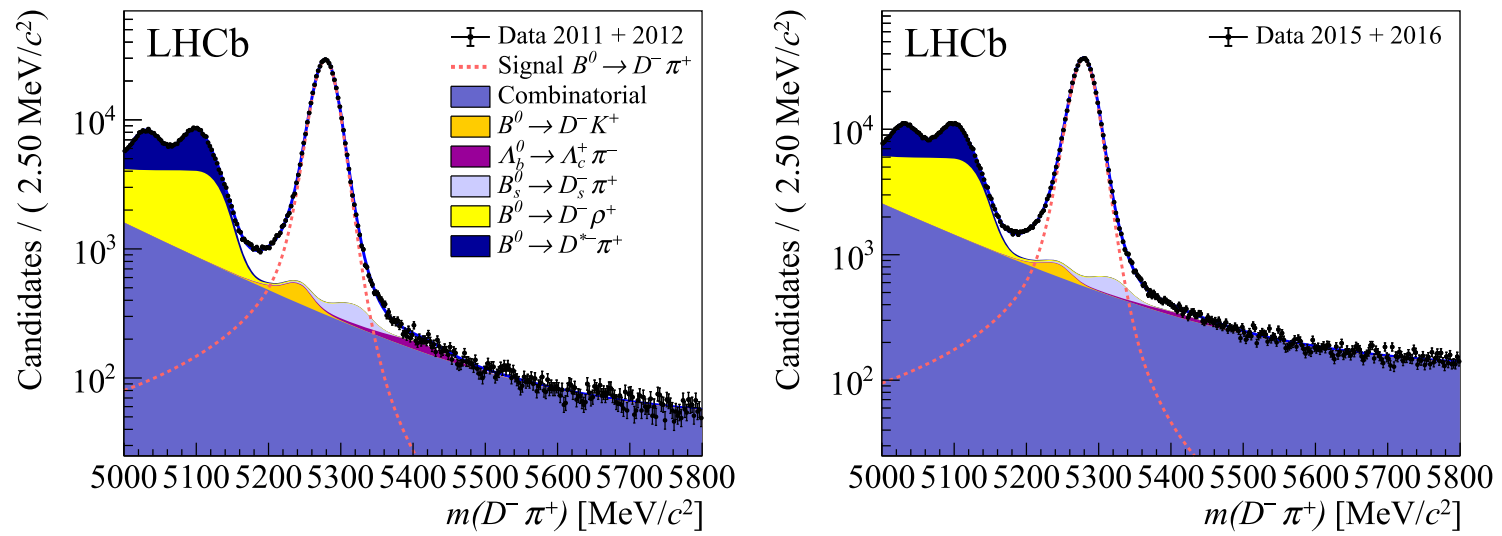

Fig. 2 The invariant mass distributions of normalisation $B^{0} \rightarrow D^{-} \pi^{+}$candidates, for (left) Run 1 and (right) Run 2 data samples. Overlaid are the fit projections along with the signal and background contributions

\section{Signal yields}

The $m\left(D^{-} \pi^{+}\right)$data distributions, with overlaid fit projections for the total, the $B^{0} \rightarrow D^{-} \pi^{+}$signal and the background components, are shown in Fig. 2. The resulting signal yields are $(4.971 \pm 0.013) \times 10^{5}$ and $(6.294 \pm 0.016) \times 10^{5}$ for Run 1 and Run 2 samples, respectively. The fit results are also used to constrain the left tail of the signal shape and the widths of the partially reconstructed backgrounds to the invariant mass distribution of $B^{0} \rightarrow D_{s}^{+} \pi^{-}$candidates.

The two-dimensional fit to $B^{0} \rightarrow D_{s}^{+} \pi^{-}$candidates is performed in the $D_{s}^{+} \pi^{-}$and $K^{+} K^{-} \pi^{+}$invariant mass distributions. The $B^{0} \rightarrow D_{s}^{+} \pi^{-}$branching fraction is determined using the yields of the signal and normalisation modes, their selection efficiencies and the known $B^{0} \rightarrow D^{-} \pi^{+}$, $D^{-} \rightarrow K^{+} \pi^{-} \pi^{-}$and $D_{s}^{+} \rightarrow K^{+} K^{-} \pi^{+}$branching fractions [38]. The two-dimensional fit is performed simultaneously for Run 1 and Run 2 data samples in which the $\mathcal{B}\left(B^{0} \rightarrow D_{s}^{+} \pi^{-}\right)$ and left-tail parameter are shared. The fit results in $B^{0} \rightarrow D_{s}^{+} \pi^{-}$ signal yields of $(8.9 \pm 0.8) \times 10^{2}$ and $(1.12 \pm 0.11) \times 10^{3}$ and $\bar{B}_{s}^{0} \rightarrow D_{s}^{+} \pi^{-}$yields of $(3.370 \pm 0.023) \times 10^{4}$ and $(4.647 \pm$ $0.027) \times 10^{4}$ for Run 1 and Run 2 samples, respectively. Figure 3 shows the $D_{s}^{+} \pi^{-}$invariant mass distributions together with the fit projections and background contributions overlaid. Additionally, the invariant mass fits to $B^{0} \rightarrow D^{-} \pi^{+}$ and $B^{0} \rightarrow D_{s}^{+} \pi^{-}$candidates are performed simultaneously to 2011,2012 and Run 2 data in order to study the collision energy dependence of $f_{s} / f_{d}$, as is described in Sect. 7.

\section{Systematic uncertainties}

Systematic uncertainties on the $\mathcal{B}\left(B^{0} \rightarrow D_{s}^{+} \pi^{-}\right)$measurement arise from choices in the fit model and the determination of trigger, BDT and PID efficiencies. Many possible sources of systematic uncertainty cancel in the ratio of either the yields or the efficiencies of $B^{0} \rightarrow D_{s}^{+} \pi^{-}$and $B^{0} \rightarrow D^{-} \pi^{+}$ events. A summary of all the systematic uncertainties is shown in Table 1. The precision of the measurement relies mostly on the accurate modelling of the signal shape and of the partially reconstructed backgrounds.

The most critical aspect of the signal shape is the description of the left tail of the $\bar{B}_{s}^{0} \rightarrow D_{s}^{+} \pi^{-}$signal, affecting the composition of signal and background around the $B^{0}$ mass. The shape of the left tail was determined from $B^{0} \rightarrow D^{-} \pi^{+}$ candidates, taking into account differences between the final states, as obtained from simulation, and was Gaussian constrained in the fit. A systematic uncertainty is assigned for the assumption of the signal shape. This is done by repeating the signal fit with a different parametrisation, i.e. the sum of a double-sided Hypatia function and a Gaussian function, which leads to a systematic uncertainty of $5.1 \%$. This parametrisation was found to be the only alternative parametrisation that satisfactorily described simulated signal candidates. Furthermore, a systematic uncertainty is assigned by fixing the mean of the $B^{0} \rightarrow D_{s}^{+} \pi^{-}$signal shape to the result of the $B^{0} \rightarrow D^{-} \pi^{+}$fit, rather than shifting by the known $B^{0}-B_{s}^{0}$ mass difference. Moreover, the width of the $B^{0} \rightarrow D_{s}^{+} \pi^{-}$signal shape is scaled by the ratio of the known $B^{0}$ and $B_{s}^{0}$ masses. The widths of the partially reconstructed backgrounds is varied by $\pm 1 \mathrm{MeV} / c^{2}$, in order to cover the differences between data and simulation as well as the differences between the $D_{s}^{+} \pi^{-}$and $D^{-} \pi^{+}$invariant mass distributions. The resulting difference between the signal yields is assigned as a systematic uncertainty.

The simulated samples are corrected for an imperfect modelling of the response of the particle identification algorithms as a function of the kinematical properties of the particle, using samples of $D^{*+}$ calibration data. A systematic uncertainty associated with the PID efficiency evaluation is assigned by varying the corrections within their uncertainties. Proton misidentification is the most difficult to control accurately from data calibration samples, as relatively little calibration data is available in the kinematic region that over- 

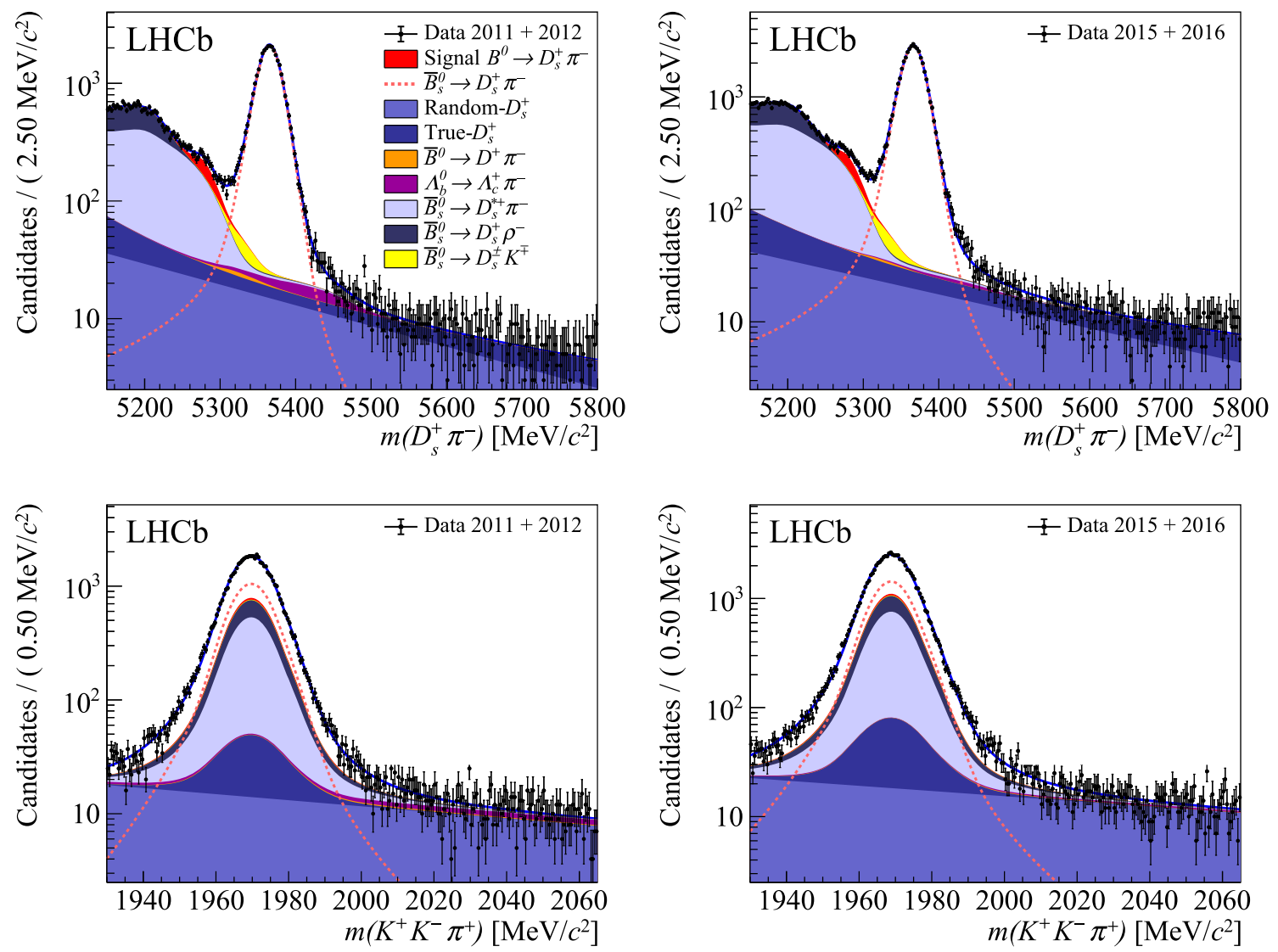

Fig. 3 The (top) $D_{s}^{+} \pi^{-}$and (bottom) $K^{+} K^{-} \pi^{+}$invariant mass distributions of signal $B^{0} \rightarrow D_{s}^{+} \pi^{-}$candidates, for (left) Run 1 and (right) Run 2 data samples. Overlaid are the fit projections along with the signal and background contributions

laps with the $B$ decay products. In addition, the Cherenkov angles of photons emitted by protons and kaons are more similar than those of kaons and pions. Thus, a systematic uncertainty is estimated from the difference between the nominal signal yields and a fit where the misidentified background $\Lambda_{b}^{0} \rightarrow \Lambda_{c}^{+} \pi^{-}$decay yield is left free to vary.

The systematic uncertainty assigned to the hardware trigger efficiency takes into account a difference in detection efficiency between kaons and pions. This mostly cancels in the ratio of $B^{0} \rightarrow D^{-} \pi^{+}$and $B^{0} \rightarrow D_{s}^{+} \pi^{-}$efficiencies, but the difference of one final-state particle is sensitive to this detection asymmetry. Moreover, an uncertainty related to the reconstruction efficiency of charged particles is taken into account, which mainly arises from the uncertainty on the $\mathrm{LHCb}$ material and the different interaction cross-section of pions and kaons with the material [44]. Additionally, a systematic uncertainty is determined on the BDT efficiency due to the difference between simulation and data. This is determined by weighting all the BDT input variables in the simulated signal sample to the signal distributions in data, which are obtained using signal weights for each candidate using the sPlot technique [45].
Table 1 Relative systematic uncertainty $\sigma$ on the $B^{0} \rightarrow D_{s}^{+} \pi^{-}$branching fraction measurement

\begin{tabular}{ll}
\hline Source & $\sigma\left(\mathcal{B}\left(B^{0} \rightarrow D_{s}^{+} \pi^{-}\right)\right)[\%]$ \\
\hline Fit model & \\
$\quad$ Signal shape parametrisation & 5.1 \\
$B^{0} \rightarrow D_{s}^{+} \pi^{-}$signal width & 1.5 \\
$B^{0} \rightarrow D_{s}^{+} \pi^{-}$mean & 0.2 \\
Partially reconstructed backgrounds & 4.2 \\
Misidentified backgrounds & 0.6 \\
Efficiencies & \\
Hardware trigger efficiency & 0.3 \\
Reconstruction efficiency & 0.5 \\
BDT efficiency & 0.7 \\
PID efficiency & 1.1 \\
Total & 6.9 \\
\hline
\end{tabular}

The systematic uncertainties on the collision energy dependence of the efficiency-corrected $\bar{B}_{s}^{0} \rightarrow D_{s}^{+} \pi^{-}$and $B^{0} \rightarrow D^{-} \pi^{+}$yield ratios are shown in Table 2 . The sources of these systematic uncertainties are the same as for the 
Table 2 Relative systematic uncertainty $\sigma$ on the ratio of the efficiencycorrected $\bar{B}_{s}^{0} \rightarrow D_{s}^{+} \pi^{-}$and $B^{0} \rightarrow D^{-} \pi^{+}$yield ratios. The ratios $\mathcal{R}_{13 \mathrm{TeV}} / \mathcal{R}_{7 \mathrm{TeV}}$ and $\mathcal{R}_{13 \mathrm{TeV}} / \mathcal{R}_{8 \mathrm{TeV}}$ are reported together as the difference of the systematic uncertainty for 7 and $8 \mathrm{TeV}$ is negligible

\begin{tabular}{lll}
\hline Source & $\sigma\left(\frac{\mathcal{R}_{13 \mathrm{TeV}}}{\mathcal{R}_{7,8 \mathrm{TeV}}}\right)[\%]$ & $\sigma\left(\frac{\mathcal{R}_{8 \mathrm{TeV}}}{\mathcal{R}_{7 \mathrm{TeV}}}\right)[\%]$ \\
\hline Fit model & & - \\
$\quad$ Signal shape parametrisation & 0.2 & - \\
$\quad$ Misidentified backgrounds & 0.2 & \\
Efficiencies & & 0.4 \\
Hardware trigger efficiency & 0.4 & 1.3 \\
BDT efficiency & 1.1 & 1.4 \\
PID efficiency & 1.4 & 2.0 \\
Total & 1.9 & \\
\hline
\end{tabular}

Table 3 Results of $B^{0} \rightarrow D_{s}^{+} \pi^{-}$and $B^{0} \rightarrow D^{-} \pi^{+}$signal efficiencies and yields, as well as the branching fractions used as input for this measurement [38]

\begin{tabular}{lll}
\hline & Run 1 & Run 2 \\
\hline$\epsilon_{B^{0} \rightarrow D_{s}^{+} \pi^{-}}(\%)$ & $0.1412 \pm 0.0010$ & $0.1922 \pm 0.0012$ \\
$\epsilon_{B^{0} \rightarrow D^{-} \pi^{+}}(\%)$ & $0.3485 \pm 0.0016$ & $0.4536 \pm 0.0016$ \\
$N_{B^{0} \rightarrow D^{-} \pi^{+}}$ & $(4.971 \pm 0.013) \times 10^{5}$ & $(6.294 \pm 0.016) \times 10^{5}$ \\
$N_{B^{0} \rightarrow D_{s}^{+} \pi^{-}}$ & $(8.9 \pm 0.8) \times 10^{2}$ & $(1.12 \pm 0.11) \times 10^{3}$ \\
\hline $\mathcal{B}\left(B^{0} \rightarrow D^{-} \pi^{+}\right)$ & $(2.52 \pm 0.13) \times 10^{-3}$ & \\
$\mathcal{B}\left(D^{-} \rightarrow K^{+} \pi^{-} \pi^{-}\right)$ & $(9.38 \pm 0.16) \times 10^{-2}$ & \\
$\mathcal{B}\left(D_{s}^{+} \rightarrow K^{+} K^{-} \pi^{+}\right)$ & $(5.39 \pm 0.15) \times 10^{-2}$ & \\
\hline
\end{tabular}

$B^{0} \rightarrow D_{s}^{+} \pi^{-}$branching fraction. Exceptions are the uncertainties on the $B^{0} \rightarrow D_{s}^{+} \pi^{-}$signal and the partially reconstructed backgrounds, which are found to be negligible, and the uncertainty on the charged-particle reconstruction efficiency, which cancels out in the double ratio of efficiencies.

\section{Results}

Table 3 gathers all measurements and inputs to determine the branching fraction according to Eq. (4). The branching fraction ratio of $B^{0} \rightarrow D_{s}^{+} \pi^{-}$and $B^{0} \rightarrow D^{-} \pi^{+}$decays is found to be

$\frac{\mathcal{B}\left(B^{0} \rightarrow D_{s}^{+} \pi^{-}\right)}{\mathcal{B}\left(B^{0} \rightarrow D^{-} \pi^{+}\right)}=(7.7 \pm 0.7 \pm 0.5 \pm 0.3) \times 10^{-3}$,

where the first uncertainty is statistical, the second systematic and the third stems from knowledge of the $D^{-} \rightarrow K^{+} \pi^{-} \pi^{-}$ and $D_{s}^{-} \rightarrow K^{-} K^{+} \pi^{-}$branching fractions.

Using the known value of $\mathcal{B}\left(B^{0} \rightarrow D^{-} \pi^{+}\right)$[38], the $B^{0} \rightarrow D_{s}^{+} \pi^{-}$branching fraction is found to be

$\mathcal{B}\left(B^{0} \rightarrow D_{s}^{+} \pi^{-}\right)=(19.4 \pm 1.8 \pm 1.3 \pm 1.2) \times 10^{-6}$, where the first uncertainty is statistical, the second systematic and the third refers to the uncertainty due to the branching fractions listed in Table 3. This result represents the most precise single measurement of $\mathcal{B}\left(B^{0} \rightarrow D_{s}^{+} \pi^{-}\right)$to date.

The $B^{0} \rightarrow D_{s}^{+} \pi^{-}$branching fraction depends on both $\left|a_{\mathrm{NF}}\right|$ and $\left|V_{u b}\right|$. Using the measurement of $\mathcal{B}\left(B^{0} \rightarrow D_{s}^{+} \pi^{-}\right)$, the product

$$
\left|V_{u b}\right|\left|a_{\mathrm{NF}}\right|=(3.14 \pm 0.20 \pm 0.25) \times 10^{-3}
$$

is obtained, where the first uncertainty is from the $B^{0} \rightarrow D_{s}^{+} \pi^{-}$branching fraction measurement and the second from the CKM and QCD parameters. The form factor $\left.F\left(B^{0} \rightarrow \pi^{-}\right)\right|_{q^{2}=m_{D_{s}^{+}}^{2}}=0.327 \pm 0.025$ is obtained using light-cone sum rules $[3,4]$ and lattice QCD calculations are used for the decay constant $f_{D_{s}^{+}}=0.2499 \pm 0.0005 \mathrm{Ge} \mathrm{V}$ [5,6]. A phase-space factor $\Phi=296.2 \pm 0.8 \mathrm{Ge} \mathrm{V}^{-2}$ is used in order to relate the branching fraction to $\left|V_{u b}\right|\left|a_{\mathrm{NF}}\right|$. Additionally, the CKM matrix element $\left|V_{c s}\right|$ is well measured and used as an input [38]. The determination of $\left|V_{u b}\right|\left|a_{\mathrm{NF}}\right|$ can be compared to the known inclusive and exclusive determinations of $\left|V_{u b}\right|$ to provide a constraint on the $\left|a_{\mathrm{NF}}\right|$ parameter as displayed in Fig. 4.

The branching fraction ratio of $B^{0} \rightarrow D_{s}^{+} \pi^{-}$and $B^{0} \rightarrow D^{-} \pi^{+}$decays can be used to determine the parameter $r_{D \pi}$, as shown in Eq. (3). Inserting the measured branching fraction ratio $\mathcal{B}\left(B^{0} \rightarrow D_{s}^{+} \pi^{-}\right) / \mathcal{B}\left(B^{0} \rightarrow D^{-} \pi^{+}\right)$, the tangent of $\theta_{c}$ [38] and the fraction between the decay constants $f_{D_{s}^{+}}$ and $f_{D^{+}}[5,6]$ into Eq. (3) gives

$r_{D \pi}=0.0163 \pm 0.0007 \pm 0.0007 \pm 0.0033$,

where the first uncertainty is statistical, the second systematic and the third arises from possible non-factorisable SU(3)breaking effects, estimated to be $20 \%$ according to Ref. [12]. SU(3)-breaking effects of about $20 \%$ are consistent with the measured $\left|a_{\mathrm{NF}}\right|$ in this analysis, see Fig. 4.

Finally, the potential dependence of the hadronisation fraction $f_{s} / f_{d}$ on collision energy is probed using the $B^{0} \rightarrow D^{-} \pi^{+}$and $\bar{B}_{s}^{0} \rightarrow D_{s}^{+} \pi^{-}$signal yields obtained in the invariant mass fits, using Eq. (5). To determine these, the fit to Run 1 data is split based on collision energy into 2011 $(7 \mathrm{TeV})$ and $2012(8 \mathrm{TeV})$, sharing the shape parameters. The measured double ratios for the different collision energies are

$$
\begin{aligned}
\mathcal{R}_{13 \mathrm{TeV}} / \mathcal{R}_{7 \mathrm{TeV}} & =1.020 \pm 0.013 \pm 0.021, \\
\mathcal{R}_{13 \mathrm{TeV}} / \mathcal{R}_{8 \mathrm{TeV}} & =1.035 \pm 0.011 \pm 0.021, \\
\mathcal{R}_{8 \mathrm{TeV}} / \mathcal{R}_{7 \mathrm{TeV}} & =0.986 \pm 0.013 \pm 0.021,
\end{aligned}
$$

where the first uncertainty is statistical and the second systematic. The average transverse momentum of the $B$ meson after full event selection is found to be 10.4, 10.6 and $10.9 \mathrm{GeV} / c$ for $p p$ collision centre-of-mass energies of 


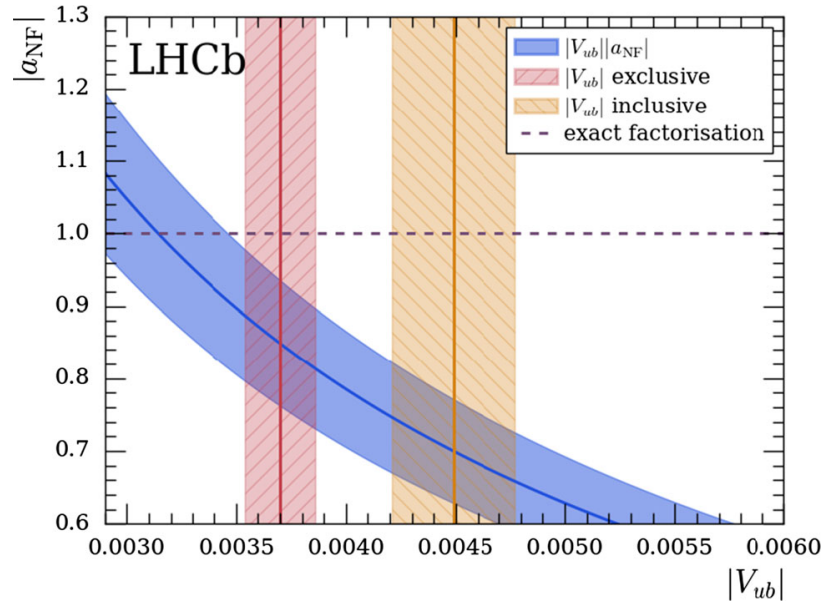

Fig. 4 Result of the determination of $\left|V_{u b}\right|\left|a_{\mathrm{NF}}\right|$. The blue line represents the result of this measurement, the vertical bands are the known exclusive and inclusive measurements of $\left|V_{u b}\right|$, which are $(3.70 \pm 0.16) \times 10^{-3}$ and $(4.49 \pm 0.28) \times 10^{-3}$, respectively [38]. The horizontal dashed line at $\left|a_{\mathrm{NF}}\right|=1.0$ represents exact factorisation. The error bands represent an uncertainty of one standard deviation

$7 \mathrm{TeV}, 8 \mathrm{TeV}$ and $13 \mathrm{TeV}$, respectively. The separate values of $\mathcal{R}$ at the three collision energies are

$$
\begin{aligned}
\mathcal{R}_{7 \mathrm{TeV}} & =0.1631 \pm 0.0018 \pm 0.0025 \pm 0.0014 \\
\mathcal{R}_{8 \mathrm{TeV}} & =0.1609 \pm 0.0013 \pm 0.0024 \pm 0.0014 \\
\mathcal{R}_{13 \mathrm{TeV}} & =0.1665 \pm 0.0011 \pm 0.0023 \pm 0.0012
\end{aligned}
$$

where the first uncertainty is statistical and the following are the uncorrelated and correlated systematic uncertainties, respectively. The value of $\mathcal{R}$ at $7 \mathrm{TeV}$ shows good agreement with the previous hadronic $f_{s} / f_{d}$ measurement at $7 \mathrm{TeV}$, which was performed using $\bar{B}_{s}^{0} \rightarrow D_{s}^{+} \pi^{-}$, $B^{0} \rightarrow D^{-} \pi^{+}$and $B^{0} \rightarrow D^{-} K^{+}$decays [46]. A visualisation of the dependence of $\mathcal{R}$ on the centre-of-mass energy is given in Fig. 5. The resulting centre-of-mass energy dependence is obtained from a linear fit using the statistical and uncorrelated systematic uncertainties and is found to be $\mathcal{R}=0.156(6)+0.0008(6) \sqrt{s}$, where $\sqrt{s}$ is in $\mathrm{TeV}$. The observed trend is in agreement with the LHCb measurement of the $f_{s} / f_{u}$ dependence upon the $p p$ collision energy [16]. The values for $\mathcal{R}$ will be used in a future work and can be used to obtain $f_{s} / f_{d}$ by correcting $\mathcal{R}$ for the relative $D$ branching fractions, the ratio of $B$ lifetimes, the form factor ratio, the contribution from non-factorisable SU(3)-breaking effects and the contribution from the exchange diagram, as given by Eq. (6).

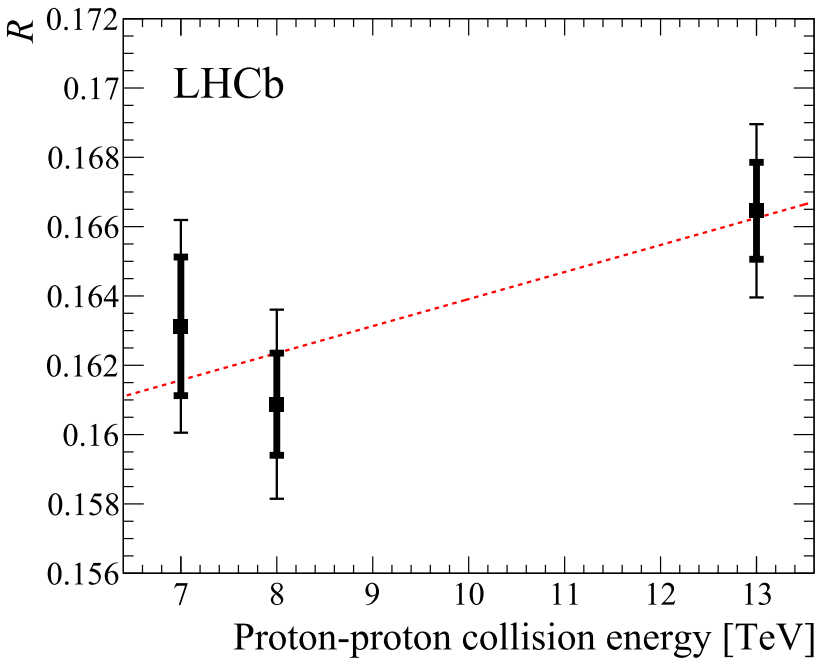

Fig. 5 Visualisation of the $p p$ collision energy dependence of the efficiency-corrected yield ratio of $\bar{B}_{s}^{0} \rightarrow D_{s}^{+} \pi^{-}$and $B^{0} \rightarrow D^{-} \pi^{+}$ decays, which scales with $f_{s} / f_{d}$. The inner error bars indicate the statistical uncertainty only, whereas the outer indicate the uncorrelated, including statistical, uncertainties. The correlated systematic uncertainty is not shown. The red dotted line represents a linear fit through the three values of $\mathcal{R}$ with uncorrelated, including statistical, uncertainties

\section{Summary}

A branching fraction measurement of the $B^{0} \rightarrow D_{s}^{+} \pi^{-}$decay is performed using $p p$ collision data taken between 2011 and 2016, leading to

$\mathcal{B}\left(B^{0} \rightarrow D_{s}^{+} \pi^{-}\right)=(19.4 \pm 1.8 \pm 1.3 \pm 1.2) \times 10^{-6}$,

where the first uncertainty is statistical, the second systematic and the third is due the branching fractions used as normalisation inputs. This is the most precise single measurement of $\mathcal{B}\left(B^{0} \rightarrow D_{s}^{+} \pi^{-}\right)$to date, and is in agreement with the current world average [38]. Using this branching fraction, the product of $\left|V_{u b}\right|$ and the non-factorisation constant $\left|a_{\mathrm{NF}}\right|$ is determined to be

$\left|V_{u b}\right|\left|a_{\mathrm{NF}}\right|=(3.14 \pm 0.20 \pm 0.25) \times 10^{-3}$.

Comparison with independently measured values of $V_{u b}$ [38] indicate that $\left|a_{\mathrm{NF}}\right|$ may deviate from unity by around $20 \%$, indicating significant non-factorisable corrections.

The measurement of the ratio of the $B^{0} \rightarrow D_{s}^{+} \pi^{-}$and $B^{0} \rightarrow D^{-} \pi^{+}$branching fractions is used to determine the $r_{D \pi}$ parameter,

$r_{D \pi}=0.0163 \pm 0.0007 \pm 0.0007 \pm 0.0033$,

where the first uncertainty is statistical, the second systematic and the third arises from possible non-factorisable SU(3)breaking effects, estimated to be $20 \%$ [12]. Knowledge of this parameter is essential to interpret the $C P$ asymmetries in $B^{0} \rightarrow D^{\mp} \pi^{ \pm}$decays. 
Finally, the efficiency-corrected yield ratio of $\bar{B}_{s}^{0} \rightarrow D_{s}^{+} \pi^{-}$and $B^{0} \rightarrow D^{-} \pi^{+}$decays, $\mathcal{R}$, is used to probe the collision energy dependence of the hadronisation fraction $f_{s} / f_{d}$.

Acknowledgements We express our gratitude to our colleagues in the CERN accelerator departments for the excellent performance of the LHC. We thank the technical and administrative staff at the LHCb institutes. We acknowledge support from CERN and from the national agencies: CAPES, CNPq, FAPERJ and FINEP (Brazil); MOST and NSFC (China); CNRS/IN2P3 (France); BMBF, DFG and MPG (Germany); INFN (Italy); NWO (Netherlands); MNiSW and NCN (Poland); MEN/IFA (Romania); MSHE (Russia); MICINN (Spain); SNSF and SER (Switzerland); NASU (Ukraine); STFC (United Kingdom); DOE NP and NSF (USA). We acknowledge the computing resources that are provided by CERN, IN2P3 (France), KIT and DESY (Germany), INFN (Italy), SURF (Netherlands), PIC (Spain), GridPP (United Kingdom), RRCKI and Yandex LLC (Russia), CSCS (Switzerland), IFIN-HH (Romania), CBPF (Brazil), PL-GRID (Poland) and OSC (USA). We are indebted to the communities behind the multiple open-source software packages on which we depend. Individual groups or members have received support from AvH Foundation (Germany); EPLANET, Marie Skłodowska-Curie Actions and ERC (European Union); A*MIDEX, ANR, Labex P2IO and OCEVU, and Région Auvergne-Rhône-Alpes (France); Key Research Program of Frontier Sciences of CAS, CAS PIFI, Thousand Talents Program, and Sci. \& Tech. Program of Guangzhou (China); RFBR, RSF and Yandex LLC (Russia); GVA, XuntaGal and GENCAT (Spain); the Royal Society and the Leverhulme Trust (United Kingdom).

Data Availability Statement This manuscript has no associated data or the data will not be deposited. [Authors' comment: All LHCb scientific output is published in journals, with preliminary results made available in Conference Reports. All are Open Access, without restriction on use beyond the standard conditions agreed by CERN. Data associated to the plots in this publication as well as in supplementary materials are made available on the CERN document server at https://cds.cern. $\mathrm{ch} /$ record/2742603. This information is taken from the LHCb External Data Access Policy which can be downloaded at http://opendata.cern. $\mathrm{ch} / \mathrm{record} / 410]$

Open Access This article is licensed under a Creative Commons Attribution 4.0 International License, which permits use, sharing, adaptation, distribution and reproduction in any medium or format, as long as you give appropriate credit to the original author(s) and the source, provide a link to the Creative Commons licence, and indicate if changes were made. The images or other third party material in this article are included in the article's Creative Commons licence, unless indicated otherwise in a credit line to the material. If material is not included in the article's Creative Commons licence and your intended use is not permitted by statutory regulation or exceeds the permitted use, you will need to obtain permission directly from the copyright holder. To view a copy of this licence, visit http://creativecomm ons.org/licenses/by/4.0/.

Funded by SCOAP ${ }^{3}$.

\section{References}

1. CKMfitter Group, J. Charles, et al., Current status of the standard model CKM fit and constraints on $\Delta F=2$ new physics. Phys. Rev. D 91, 073007 (2015). arXiv:1501.05013, updated results and plots available at http://www.ckmfitter.in2p3.fr/
2. UTfit Collaboration, M. Bona, et al., The unitarity triangle fit in the standard model and hadronic parameters from lattice QCD: a reappraisal after the measurements of $\Delta m_{s}$ and $B R\left(B \rightarrow \tau \nu_{\tau}\right)$. JHEP 10, 081 (2006). arXiv:hep-ph/0606167, updated results and plots available at http://www.utfit.org/

3. P. Ball, R. Zwicky, New results on $B \rightarrow \pi, K, \eta$ decay form factors from light-cone sum rules. Phys. Rev. D 71, 014015 (2005). arXiv:hep-ph/0406232

4. P. Ball, $\left|V_{u b}\right|$ from UTangles and $B^{0} \rightarrow \pi^{-} \ell^{+} v$. Phys. Lett. B 644 , 38 (2007). arXiv:hep-ph/0611108

5. A. Bazavov et al., $B$ - and $D$-meson leptonic decay constants from four-flavor lattice QCD. Phys. Rev. D 98, 074512 (2018). arXiv: 1712.09262

6. N. Carrasco et al., Leptonic decay constants $f_{K}, f_{D}$, and $f_{D_{s}}$ with $N_{f}=2+1+1$ twisted-mass lattice QCD. Phys. Rev. D 91, 054507 (2015). arXiv:1411.7908

7. M. Beneke, G. Buchalla, M. Neubert, C.T. Sachrajda, QCD factorization for exclusive, nonleptonic B meson decays: general arguments and the case of heavy light final states. Nucl. Phys. B 591, 313 (2000). arXiv:hep-ph/0006124

8. BaBar Collaboration, B. Aubert, et al., Measurement of time-dependent $\mathrm{CP}$-violating asymmetries and constraints on $\sin (2 \beta+\gamma)$ with partial reconstruction of $B \rightarrow D^{* \mp} \pi^{ \pm}$decays. Phys. Rev. D 71, 112003 (2005). arXiv:hep-ex/0504035

9. BaBar Collaboration, B. Aubert, et al., Measurement of timedependent CP asymmetries in $B^{0} \rightarrow D^{(*) \pm} \pi^{\mp}$ and $B^{0} \rightarrow D^{ \pm} \rho^{\mp}$ decays. Phys. Rev. D 73, 111101 (2006). arXiv:hep-ex/0602049

10. Belle Collaboration, F.J. Ronga, et al., Measurements of CP violation in $B^{0} \rightarrow D^{*-} \pi^{+}$and $B^{0} \rightarrow D^{-} \pi^{+}$decays. Phys. Rev. D 73, 092003 (2006). arXiv:hep-ex/0604013

11. Belle Collaboration, S. Bahinipati, et al., Measurements of timedependent $\mathrm{CP}$ asymmetries in $B \rightarrow D^{* \mp} \pi^{ \pm}$decays using a partial reconstruction technique. Phys. Rev. D 84, 021101 (2011). arXiv: 1102.0888

12. K. De Bruyn et al., Exploring $B_{s} \rightarrow D_{s}^{(*) \pm} K^{\mp}$ decays in the presence of a sizable width difference $\Delta \Gamma_{s}$. Nucl. Phys. B 868, 351 (2013). arXiv:1208.6463

13. LHCb Collaboration, R. Aaij, et al., Measurement of $C P$ violation in $B^{0} \rightarrow D^{ \pm} \pi^{\mp}$ decays. JHEP 06, 084 (2018). arXiv: 1805.03448

14. BaBar Collaboration, B. Aubert, et al., Measurement of the branching fractions of the rare decays $B^{0} \rightarrow D_{s}^{(*)+} \pi^{-}, B^{0} \rightarrow$ $D_{s}^{(*)+} \rho^{-}$, and $B^{0} \rightarrow D_{s}^{(*)-} K^{(*)+}$. Phys. Rev. D 78, 032005 (2008). arXiv:0803.4296

15. Belle Collaboration, A. Das, et al., Measurements of branching fractions for $B^{0} \rightarrow D_{s}^{+} \pi^{-} v$ and $\bar{B}^{0} \rightarrow D_{s}^{+} K^{-}$. Phys. Rev. D 82, 051103 (2010). arXiv: 1007.4619

16. LHCb Collaboration, R. Aaij, et al., Measurement of $f_{s} / f_{u}$ variation with proton-proton collision energy and $B$-meson kinematics. Phys. Rev. Lett. 124, 122002 (2020). arXiv:1910.09934

17. LHCb Collaboration, R. Aaij, et al., Measurement of the $B_{s}^{0} \rightarrow \mu^{+} \mu^{-}$branching fraction and effective lifetime and search for $B^{0} \rightarrow \mu^{+} \mu^{-}$decays. Phys. Rev. Lett. 118, 191801 (2017). arXiv: 1703.05747

18. R. Fleischer, N. Serra, N. Tuning, Tests of factorization and SU(3) relations in B decays into heavy-light final states. Phys. Rev. D 83, 014017 (2011). arXiv: 1012.2784

19. LHCb Collaboration, A.A. Alves Jr., et al., The LHCb detector at the LHC. JINST 3, S08005 (2008)

20. LHCb Collaboration, R. Aaij, et al., LHCb detector performance. Int. J. Mod. Phys. A 30, 1530022 (2015). arXiv:1412.6352

21. R. Aaij et al., Performance of the LHCb Vertex Locator. JINST 9 , P09007 (2014). arXiv: 1405.7808

22. R. Arink et al., Performance of the LHCb Outer Tracker. JINST 9, P01002 (2014). arXiv:1311.3893 
23. P. d'Argent et al., Improved performance of the $\mathrm{LHCb}$ Outer Tracker in LHC Run 2. JINST 12, P11016 (2017). arXiv: 1708.00819

24. M. Adinolfi et al., Performance of the LHCb RICH detector at the LHC. Eur. Phys. J. C 73, 2431 (2013). arXiv:1211.6759

25. A.A. Alves Jr. et al., Performance of the LHCb muon system. JINST 8, P02022 (2013). arXiv:1211.1346

26. R. Aaij et al., The LHCb trigger and its performance in 2011. JINST 8, P04022 (2013). arXiv:1211.3055

27. T. Sjöstrand, S. Mrenna, P. Skands, A brief introduction to PYTHIA 8.1. Comput. Phys. Commun. 178, 852 (2008). arXiv:0710.3820

28. I. Belyaev et al., Handling of the generation of primary events in Gauss, the LHCb simulation framework. J. Phys. Conf. Ser. 331, 032047 (2011)

29. D.J. Lange, The EvtGen particle decay simulation package. Nucl. Instrum. Methods A 462, 152 (2001)

30. P. Golonka, Z. Was, PHOTOS Monte Carlo: a precision tool for QED corrections in $Z$ and $W$ decays. Eur. Phys. J. C 45, 97 (2006). arXiv:hep-ph/0506026

31. Geant4 Collaboration, J. Allison, et al., Geant 4 developments and applications. IEEE Trans. Nucl. Sci. 53, 270 (2006)

32. Geant4 Collaboration, S. Agostinelli, et al., Geant4: a simulation toolkit. Nucl. Instrum. Methods A 506, 250 (2003)

33. M. Clemencic et al., The LHCb simulation application, Gauss: Design, evolution and experience. J. Phys. Conf. Ser. 331, 032023 (2011). arXiv:1210.6861

34. V.V. Gligorov, M. Williams, Efficient, reliable and fast high-level triggering using a bonsai boosted decision tree. JINST 8, P02013 (2013). arXiv:1210.6861

35. L. Breiman, J.H. Friedman, R.A. Olshen, C.J. Stone, Classification and Regression Trees (Wadsworth international group, Belmont, 1984)
36. B.P. Roe et al., Boosted decision trees, an alternative to artificial neural networks. Nucl. Instrum. Methods A543, 577 (2005). arXiv:physics/0408124

37. U.P. Eitschberger, Flavour-tagged measurement of $\mathrm{CP}$ observables in $B_{s}^{0} \rightarrow D_{s}^{\mp} K^{ \pm}$decays with the LHCb experiment. Ph.D. thesis, Tech. U., Dortmund (main) (2018). https://doi.org/10.17877/ DE290R-18881

38. Particle Data Group, P.A. Zyla, et al., Review of particle physics. Prog. Theor. Exp. Phys. 2020, 083 C01 (2020)

39. LHCb Collaboration, R. Aaij, et al., Measurement of $C P$ asymmetry in $B_{s}^{0} \rightarrow D_{s}^{\mp} K^{ \pm}$decays. JHEP 03, 059 (2018). arXiv: 1712.07428

40. D. Martínez Santos, F. Dupertuis, Mass distributions marginalized over per-event errors. Nucl. Instrum. Methods A 764, 150 (2014). arXiv: 1312.5000

41. N.L. Johnson, Systems of frequency curves generated by methods of translation. Biometrika 36, 149 (1949)

42. LHCb Collaboration, R. Aaij, et al., Measurement of $C P$ observables in $B^{ \pm} \rightarrow D^{(*)} K^{ \pm}$and $B^{ \pm} \rightarrow D^{(*)} \pi^{ \pm}$decays. Phys. Lett. B 777, 16 (2018). arXiv: 1708.06370

43. K.S. Cranmer, Kernel estimation in high-energy physics. Comput. Phys. Commun. 136, 198 (2001). arXiv:hep-ex/0011057

44. LHCb Collaboration, R. Aaij, et al., Measurement of the branching fraction and $C P$ asymmetry in $B^{+} \rightarrow J / \pi \rho^{+}$decays. Eur. Phys. J. C 79, 537 (2019). arXiv:1812.07041

45. M. Pivk, F.R. Le Diberder, sPlot: a statistical tool to unfold data distributions. Nucl. Instrum. Methods A 555, 356 (2005). arXiv:physics/0402083

46. LHCb Collaboration, R. Aaij, et al., Measurement of the fragmentation fraction ratio $f_{s} / f_{d}$ and its dependence on $B$ meson kinematics. JHEP 04, 001 (2013). arXiv:1301.5286 


\section{LHCb Collaboration}

R. Aaij $^{32}$, C. Abellán Beteta ${ }^{50}$, T. Ackernley ${ }^{60}$, B. Adeva $^{46}$, M. Adinolf ${ }^{54}$, H. Afsharnia ${ }^{9}$, C. A. Aidala ${ }^{85}$, S. Aiola ${ }^{25}$, Z. Ajaltouni ${ }^{9}$, S. Akar ${ }^{65}$, J. Albrecht ${ }^{15}$, F. Alessio ${ }^{48}$, M. Alexander ${ }^{59}$, A. Alfonso Albero ${ }^{45}$, Z. Aliouche ${ }^{62}$, G. Alkhazov ${ }^{38}$, P. Alvarez Cartelle ${ }^{55}$, S. Amato ${ }^{2}$, Y. Amhis ${ }^{11}$, L. An ${ }^{48}$, L. Anderlinii ${ }^{22}$, A. Andreianov ${ }^{38}$, M. Andreotti ${ }^{21}$, F. Archilli ${ }^{17}$, A. Artamonov ${ }^{44}, \quad$ M. Artuso ${ }^{68}, \quad$ K. Arzymatov ${ }^{42}, \quad$ E. Aslanides ${ }^{10}, \quad$ M. Atzeni ${ }^{50}, \quad$ B. Audurier ${ }^{12}, \quad$ S. Bachmann ${ }^{17}$, M. Bachmayer ${ }^{49}$, J. J. Back ${ }^{56}$, S. Baker ${ }^{61}$, P. Baladron Rodriguez ${ }^{46}$, V. Balagura ${ }^{12}$, W. Baldini ${ }^{21}$, J. Baptista Leite $^{1}$, R. J. Barlow ${ }^{62}$, S. Barsuk ${ }^{11}$, W. Barter ${ }^{61}$, M. Bartolini' ${ }^{2, g}$, F. Baryshnikov ${ }^{82}$, J. M. Basels ${ }^{14}$, G. Bassi' ${ }^{29}$, B. Batsukh ${ }^{68}$, A. Battig ${ }^{15}$, A. Bay ${ }^{49}$, M. Becker ${ }^{15}$, F. Bedeschi ${ }^{29}$, I. Bediaga ${ }^{1}$, A. Beiter ${ }^{68}$, V. Belavin ${ }^{42}$, S. Belinn ${ }^{27}$, V. Bellee ${ }^{49}$, K. Belous ${ }^{44}$, I. Belov ${ }^{40}$, I. Belyaev ${ }^{41}$, G. Bencivenni' ${ }^{23}$, E. Ben-Haim ${ }^{13}$, A. Berezhnoy ${ }^{40}$, R. Bernet ${ }^{50}$, D. Berninghoff ${ }^{17}$, H. C. Bernstein ${ }^{68}$, C. Bertella ${ }^{48}$, A. Bertolin ${ }^{28}$, C. Betancourt ${ }^{50}$, F. Betti ${ }^{20, c}$, Ia. Bezshyiko ${ }^{50}$, S. Bhasin ${ }^{54}$, J. Bhom ${ }^{35}$, L. Bian ${ }^{73}$, M. S. Bieker ${ }^{15}$, S. Bifani ${ }^{53}$, P. Billoir ${ }^{13}, \quad$ M. Birch ${ }^{61}$, F. C. R. Bishop ${ }^{55}$, A. Bizzeti $22, j, \quad$ M. Bjørn ${ }^{63}$, M. P. Blago ${ }^{48}$, T. Blake ${ }^{56}$, F. Blanc ${ }^{49}$, S. Blusk ${ }^{68}$, D. Bobulska ${ }^{59}$, J. A. Boelhauve ${ }^{15}$, O. Boente Garcia ${ }^{46}$, T. Boettcher ${ }^{64}$, A. Boldyrev ${ }^{81}$, A. Bondar ${ }^{43}$, N. Bondar ${ }^{38}$, S. Borghi ${ }^{62}$, M. Borisyak ${ }^{42}$, M. Borsato ${ }^{17}$, J. T. Borsuk ${ }^{35}$, S. A. Bouchiba ${ }^{49}$, T. J. V. Bowcock ${ }^{60}$, A. Boyer ${ }^{48}$ ， C. Bozzi ${ }^{21}$, M. J. Bradley ${ }^{61}$ ， S. Braun ${ }^{66}$, A. Brea Rodriguez ${ }^{46}$, M. Brodski ${ }^{48}$,

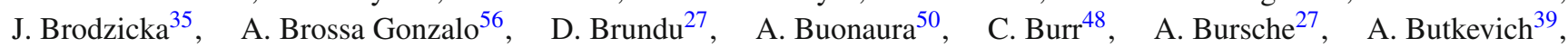
J. S. Butter ${ }^{32}$ (1) J. Buytaert ${ }^{48}$, W. Byczynski ${ }^{48}$, S. Cadeddu ${ }^{27}$, H. Cai ${ }^{73}$, R. Calabrese ${ }^{21, e}$, L. Calefice ${ }^{13,15}$, L. Calero Diaz ${ }^{23}$, S. Cali ${ }^{23}$, R. Calladine ${ }^{53}$, M. Calvi ${ }^{26, i}$, M. Calvo Gomez ${ }^{84}$, P. Camargo Magalhaes ${ }^{54}$, A. Cambonii ${ }^{45,84}$, P. Campana ${ }^{23}$, A. F. Campoverde Quezada ${ }^{6}$, S. Capelli ${ }^{26, i}$, L. Capriotti ${ }^{20, c}$, A. Carbone ${ }^{20, c}$, G. Carboni ${ }^{31}$, R. Cardinale ${ }^{24, g}$, A. Cardini ${ }^{27}$, I. Carli ${ }^{4}$, P. Carniti ${ }^{26, i}$, K. Carvalho Akiba ${ }^{32}$, A. Casais Vidal ${ }^{46}$, G. Casse ${ }^{60}$, M. Cattaneo ${ }^{48}$, G. Cavallero ${ }^{48}$, S. Celani ${ }^{49}$, J. Cerasoli ${ }^{10}$, A. J. Chadwick ${ }^{60}$, M. G. Chapman ${ }^{54}$, M. Charles ${ }^{13}$, Ph. Charpentier ${ }^{48}$, G. Chatzikonstantinidis ${ }^{53}$, C. A. Chavez Barajas ${ }^{60}$, M. Chefdeville ${ }^{8}$, C. Chen ${ }^{3}$, S. $\mathrm{Chen}^{27}$, A. Chernov ${ }^{35}$, S.-G. Chitic ${ }^{48}$, V. Chobanova ${ }^{46}$, S. Cholak ${ }^{49}$,

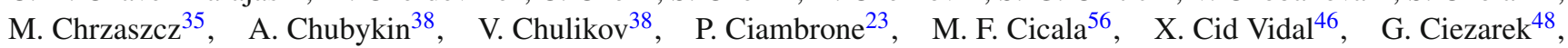
P. E. L. Clarke ${ }^{58}$, M. Clemencic ${ }^{48}$, H. V. Cliff ${ }^{55}$, J. Closier ${ }^{48}$, J. L. Cobbledick ${ }^{62}, \quad$ V. Coco ${ }^{48}$, J. A. B. Coelho ${ }^{11}$, J. Cogan ${ }^{10}$, E. Cogneras ${ }^{9}$, L. Cojocariu ${ }^{37}$, P. Collins ${ }^{48}$, T. Colombo ${ }^{48}$, L. Congedo ${ }^{19, b}$, A. Contu ${ }^{27}$, N. Cooke ${ }^{53}$, G. Coombs ${ }^{59}$, G. Corti ${ }^{48}$, C. M. Costa Sobral ${ }^{56}$, B. Couturier ${ }^{48}$, D. C. Craik ${ }^{64}$, J. Crkovská ${ }^{67}$, M. Cruz Torres ${ }^{1}$, R. Currie ${ }^{58}$, C. L. Da Silva ${ }^{67}$, E. Dall'Occo ${ }^{15}$, J. Dalseno ${ }^{46}$, C. D’Ambrosio ${ }^{48}$, A. Danilina ${ }^{41}$, P. d'Argent ${ }^{48}$, A. Davis ${ }^{62}$, O. De Aguiar Francisco ${ }^{62}$, K. De Bruyn ${ }^{78}$, S. De Capua ${ }^{62}$, M. De Cian ${ }^{49}$, J. M. De Miranda ${ }^{1}$,L. De Paula ${ }^{2}$, M. De Serio ${ }^{19, b}$, D. De Simone ${ }^{50}$, P. De Simone ${ }^{23}$, J. A. de Vries ${ }^{79}$, C. T. Dean ${ }^{67}$, D. Decamp ${ }^{8}$, L. Del Buono ${ }^{13}$, B. Delaney ${ }^{55}$, H.-P. Dembinski ${ }^{15}$, A. Dendek ${ }^{34}$, V. Denysenko ${ }^{50}$, D. Derkach ${ }^{81}$, O. Deschamps 9 , F. Desse ${ }^{11}$, F. Dettori ${ }^{27, d}$, B. Dey ${ }^{73}$, P. Di Nezza ${ }^{23}$, S. Didenko ${ }^{82}$, L. Dieste Maronas ${ }^{46}$, H. Dijkstra ${ }^{48}$, V. Dobishuk ${ }^{52}$, A. M. Donohoe ${ }^{18}$, F. Dordei ${ }^{27}$, A. C. $\operatorname{dos}_{\text {Reis }}{ }^{1}, \quad$ L. Douglas ${ }^{59}$, A. Dovbnya ${ }^{51}, \quad$ A. G. Downes ${ }^{8}, \quad$ K. Dreimanis ${ }^{60}, \quad$ M. W. Dudek ${ }^{35}$, L. Dufour ${ }^{48}$, V. Duk ${ }^{77}$, P. Durante ${ }^{48}$, J. M. Durham ${ }^{67}$, D. Dutta ${ }^{62}$, M. Dziewiecki ${ }^{17}, \quad$ A. Dziurda ${ }^{35}$, A. Dzyuba ${ }^{38}$, S. Easo ${ }^{57}$, U. Egede ${ }^{69}$, V. Egorychev ${ }^{41}$, S. Eidelman ${ }^{43, u}$, S. Eisenhardt ${ }^{58}$, S. Ek-In ${ }^{49}$, L. Eklund ${ }^{59, v}$, S. Ely ${ }^{68}$, A. Ene ${ }^{37}$, E. Epple ${ }^{67}$, S. Escher ${ }^{14}$, J. Eschle ${ }^{50}$, S. Esen ${ }^{32}$, T. Evans ${ }^{48}$, A. Falabella ${ }^{20}$, J. Fan ${ }^{3}$, Y. Fan ${ }^{6}$, B. Fang ${ }^{73}$, N. Farley ${ }^{53}$, S. Farry ${ }^{60}$, D. Fazzini ${ }^{26, i}$, P. Fedin ${ }^{41}$, M. Féo ${ }^{48}$, P. Fernandez Declara ${ }^{48}$, A. Fernandez Prieto ${ }^{46}$, J. M. Fernandez-tenllado Arribas ${ }^{45}$, F. Ferrari $^{20, c}$, L. Ferreira Lopes ${ }^{49}$, F. Ferreira Rodrigues ${ }^{2}$, S. Ferreres Sole ${ }^{32}$, M. Ferrillo ${ }^{50}$, M. Ferro-Luzzi ${ }^{48}$, S. Filippov $^{39}$, R. A. Fini ${ }^{19}$, M. Fiorini ${ }^{21, e}$, M. Firlej ${ }^{34}$, K. M. Fischer ${ }^{63}$, C. Fitzpatrick ${ }^{62}$, T. Fiutowski ${ }^{34}$, F. Fleuret ${ }^{12}$, M. Fontana ${ }^{13}$, F. Fontanelli24,g, R. Forty ${ }^{48}$, V. Franco Lima ${ }^{60}$, M. Franco Sevilla ${ }^{66}$, M. Frank ${ }^{48}$, E. Franzoso ${ }^{21}$, G. Frau ${ }^{17}$, C. Frei ${ }^{48}$, D. A. Friday ${ }^{59}$, J. Fu ${ }^{25}$, Q. Fuehring ${ }^{15}$, W. Funk ${ }^{48}$, E. Gabriel ${ }^{32}$, T. Gaintseva ${ }^{42}$, A. Gallas Torreira ${ }^{46}$, D. Galli ${ }^{20, \mathrm{c}}$, S. Gambetta ${ }^{48,58}$, Y. Gan ${ }^{3}$, M. Gandelman ${ }^{2}$, P. Gandini' ${ }^{25}$, Y. Gao ${ }^{5}$, M. Garau ${ }^{27}$, L. M. Garcia Martin ${ }^{56}$, $\begin{array}{lll}\text { P. Garcia Moreno } & 45\end{array}$ J. García Pardiñas ${ }^{26}$, B. Garcia Plana ${ }^{46}$, F. A. Garcia Rosales ${ }^{12}$, L. Garrido ${ }^{45}$, C. Gaspar ${ }^{48}$, R. E. Geertsema ${ }^{32}$, D. Gerick ${ }^{17}$, L. L. Gerken ${ }^{15}$, E. Gersabeck ${ }^{62}, \quad$ M. Gersabeck ${ }^{62}, \quad$ T. Gershon ${ }^{56}, \quad$ D. Gerstel ${ }^{10}$, Ph. Ghez ${ }^{8}$, V. Gibson ${ }^{55}$, M. Giovannetti ${ }^{23, o}$, A. Gioventù ${ }^{46}$, P. Gironella Gironell ${ }^{45}$, L. Giubega ${ }^{37}$, C. Giugliano ${ }^{21, e, 48}$, K. Gizdov ${ }^{58}$, E. L. Gkougkousis ${ }^{48}$, V. V. Gligorov ${ }^{13}$, C. Göbel ${ }^{70}$, E. Golobardes ${ }^{84}$, D. Golubkov ${ }^{41}$, A. Golutvin ${ }^{61,82}$, A. Gomes ${ }^{1, \text { a }}$, S. Gomez Fernandez ${ }^{45}$, F. Goncalves Abrantes ${ }^{70}$, M. Goncerz ${ }^{35}$, G. Gong ${ }^{3}$, P. Gorbounov ${ }^{41}$, I. V. Gorelov ${ }^{40}$, C. Gotti ${ }^{26, i}$, E. Govorkova ${ }^{48}$, J. P. Grabowski ${ }^{17}$, R. Graciani Diaz ${ }^{45}$, T. Grammatico ${ }^{13}$, L. A. Granado Cardoso ${ }^{48}$, E. Graugés ${ }^{45}$, E. Graverini ${ }^{49}$, G. Graziani ${ }^{22}$, A. Grecu ${ }^{37}$, L. M. Greeven ${ }^{32}$, P. Griffith ${ }^{21, e}$, L. Grillo ${ }^{62}$, S. Gromov ${ }^{82}$, B. R. Gruberg Cazon ${ }^{63}$, C. Gu ${ }^{3}$, M. Guarise ${ }^{21}$, P. A. Günther ${ }^{17}$, E. Gushchin ${ }^{39}$, A. Guth ${ }^{14}$, Y. Guz ${ }^{44,48}$, T. Gys ${ }^{48}$, T. Hadavizadeh ${ }^{69}$, G. Haefeli ${ }^{49}$, C. Haen ${ }^{48}$, J. Haimberger ${ }^{48}$, T. Halewood-leagas ${ }^{60}$, P. M. Hamilton ${ }^{66}$, Q. $\operatorname{Han}^{7}$, X. $\operatorname{Han}^{17}$, T. H. Hancock ${ }^{63}$, S. Hansmann-Menzemer ${ }^{17}$, N. Harnew ${ }^{63}$, T. Harrison ${ }^{60}$, C. Hasse ${ }^{48}$, M. Hatch ${ }^{48}$, J. He ${ }^{6}$, M. Hecker ${ }^{61}$, K. Heijhoff ${ }^{32}$, K. Heinicke ${ }^{15}$, A. M. Hennequin ${ }^{48}$, K. Hennessy ${ }^{60}$, L. Henry ${ }^{25,47}$, J. Heuel ${ }^{14}$, A. Hicheur ${ }^{2}$, D. Hill ${ }^{49}$, M. Hilton ${ }^{62}$, S. E. Hollitt ${ }^{15}$, J. Hu ${ }^{72}$, J. Hu ${ }^{17}$, W. Hu ${ }^{7}$, W. Huang ${ }^{6}$, X. Huang ${ }^{73}$, W. Hulsbergen ${ }^{32}$, R. J. Hunter ${ }^{56}$, 


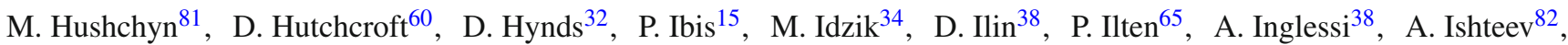
K. Ivshin ${ }^{38}$, R. Jacobsson ${ }^{48}$, S. Jakobsen ${ }^{48}$, E. Jans ${ }^{32}$, B. K. Jashal ${ }^{47}$ ， A. Jawahery ${ }^{66}$ ， V. Jevtic ${ }^{15}$, M. Jezabek ${ }^{35}$, F. Jiang ${ }^{3}$ ， M. John ${ }^{63}$ ， D. Johnson ${ }^{48}$ ， C. R. Jones ${ }^{55}$ ， T. P. Jones ${ }^{56}$ ， B. Jost ${ }^{48}$ ， N. Jurik ${ }^{48}$ ， S. Kandybei ${ }^{51}$ ， Y. Kang ${ }^{3}$, M. Karacson ${ }^{48}$, N. Kazeev ${ }^{81}$, F. Keizer ${ }^{48,55}$, M. Kenzie ${ }^{56}$, T. Ketel ${ }^{33}$, B. Khanji ${ }^{15}$, A. Kharisova ${ }^{83}$, S. Kholodenko ${ }^{44}$, K. E. Kim ${ }^{68}$, T. Kirn ${ }^{14}$, V. S. Kirsebom ${ }^{49}$, O. Kitouni ${ }^{64}$, S. Klaver ${ }^{32}$, K. Klimaszewski ${ }^{36}$, S. Koliiev ${ }^{52}$, A. Kondybayeva ${ }^{82}$, A. Konoplyannikov ${ }^{41}$, P. Kopciewicz ${ }^{34}$, R. Kopecna ${ }^{17}$, P. Koppenburg ${ }^{32}$, M. Korolev ${ }^{40}$, I. Kostiuk ${ }^{32,52}$, O. Kot ${ }^{52}$, S. Kotriakhova ${ }^{30,38}$, P. Kravchenko ${ }^{38}$, L. Kravchuk ${ }^{39}$ ，R. D. Krawczyk ${ }^{48}$ ，M. Kreps ${ }^{56}$, F. Kress ${ }^{61}$, S. Kretzschmar ${ }^{14}$, P. Krokovny ${ }^{43, u}$, W. Krupa ${ }^{34}$, W. Krzemien ${ }^{36}$, W. Kucewicz ${ }^{35, \text { s, M. Kucharczyk }}{ }^{35}$, V. Kudryavtsev ${ }^{43, u}$, H. S. Kuindersma ${ }^{32}$, G. J. Kunde ${ }^{67}$, T. Kvaratskheliya ${ }^{41}$, D. Lacarrere ${ }^{48}$, G. Lafferty ${ }^{62}$, A. Lai ${ }^{27}$, A. Lampis ${ }^{27}$, D. Lancierini ${ }^{50}$, J. J. Lane ${ }^{62}$, R. Lane ${ }^{54}$, G. Lanfranchi ${ }^{23}$, C. Langenbruch ${ }^{14}$, J. Langer ${ }^{15}$, O. Lantwin ${ }^{50,82}$, T. Latham ${ }^{56}$, F. Lazzari ${ }^{29, p}$, R. Le Gac ${ }^{10}$,

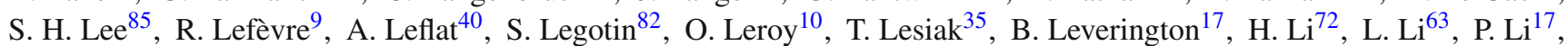

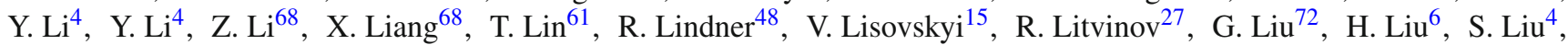

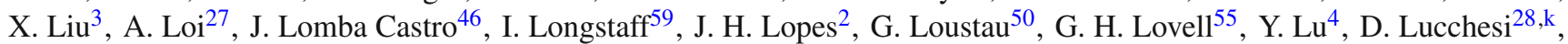
S. Luchuk ${ }^{39}$, M. Lucio Martinez ${ }^{32}$, V. Lukashenko ${ }^{32}$, Y. Luo ${ }^{3}$, A. Lupato ${ }^{62}$, E. Luppi ${ }^{21, e}$, O. Lupton ${ }^{56}$, A. Lusiani ${ }^{29,1}$, X. Lyu ${ }^{6}$, L. Ma ${ }^{4}$, S. Maccolini ${ }^{20, c}$, F. Machefert ${ }^{11}$, F. Maciuc ${ }^{37}$, V. Macko ${ }^{49}$, P. Mackowiak ${ }^{15}$, S. Maddrell-Mander ${ }^{54}$, O. Madejczyk ${ }^{34}$, L. R. Madhan Mohan ${ }^{54}$, O. Maev ${ }^{38}$, A. Maevskiy ${ }^{81}$, D. Maisuzenko ${ }^{38}$, M. W. Majewski ${ }^{34}$, S. Malde ${ }^{63}$, B. Malecki ${ }^{48}$, A. Malinin ${ }^{80}$, T. Maltsev ${ }^{43, u}$, H. Malygina ${ }^{17}$, G. Manca ${ }^{27, d}$, G. Mancinellii ${ }^{10}$, R. Manera Escalero ${ }^{45}$, D. Manuzzi ${ }^{20, c}$, D. Marangotto ${ }^{25, h}$, J. Maratas ${ }^{9, r}$, J. F. Marchand ${ }^{8}$, U. Marconi ${ }^{20}$, S. Mariani ${ }^{22, f, 48}$, C. Marin Benito ${ }^{11}$, M. Marinangeli ${ }^{49}$, P. Marino ${ }^{49,1}$, J. Marks ${ }^{17}$, P. J. Marshall ${ }^{60}$, G. Martellotti ${ }^{30}$, L. Martinazzoli ${ }^{48, i}$, M. Martinelli ${ }^{26, i}$, D. Martinez Santos ${ }^{46}$, F. Martinez Vidal ${ }^{47}$, A. Massafferri ${ }^{1}$, M. Materok ${ }^{14}$, R. Matev ${ }^{48}$, A. Mathad ${ }^{50}$, Z. Mathe ${ }^{48}$, V. Matiunin ${ }^{41}$, C. Matteuzzi ${ }^{26}$, K. R. Mattioli ${ }^{85}$, A. Mauri ${ }^{32}$, E. Maurice ${ }^{12}$, J. Mauricio ${ }^{45}$, M. Mazurek ${ }^{36}$, M. McCann ${ }^{61}$, L. Mcconnell ${ }^{18}$, T. H. Mcgrath ${ }^{62}$, A. McNab ${ }^{62}$, R. McNulty ${ }^{18}$, J. V. Mead $^{60}$, B. Meadows ${ }^{65}$, C. Meaux ${ }^{10}$, G. Meier ${ }^{15}$, N. Meinert ${ }^{76}$, D. Melnychuk ${ }^{36}$, S. Meloni ${ }^{26, i}$, M. Merk ${ }^{32,79}$, A. Merli ${ }^{25}$, L. Meyer Garcia ${ }^{2}, \quad$ M. Mikhasenko ${ }^{48}$, D. A. Milanes ${ }^{74}$, E. Millard ${ }^{56}$, M. Milovanovic ${ }^{48}$, M.-N. Minard ${ }^{8}$, L. Minzoni ${ }^{21, e}$, S. E. Mitchell ${ }^{58}$, B. Mitreska ${ }^{62}$, D. S. Mitzel ${ }^{48}$, A. Mödden ${ }^{15}$, R. A. Mohammed ${ }^{63}$, R. D. Moise ${ }^{61}$, T. Mombächer ${ }^{15}$, I. A. Monroy ${ }^{74}$, S. Monteil ${ }^{9}$, M. Morandin ${ }^{28}$, G. Morello ${ }^{23}$, M. J. Morello ${ }^{29,1}$, J. Moron ${ }^{34}$, A. B. Morris ${ }^{75}$, A. G. Morris ${ }^{56}$, R. Mountain ${ }^{68}$, H. $\mathrm{Mu}^{3}$, F. Muheim ${ }^{58}$, M. Mukherjee ${ }^{7}$, M. Mulder ${ }^{48}$, D. Müller ${ }^{48}$, K. Müller ${ }^{50}$, C. H. Murphy ${ }^{63}$, D. Murray ${ }^{62}$, P. Muzzetto ${ }^{27,48}$, P. Naik ${ }^{54}$, T. Nakada ${ }^{49}$, R. Nandakumar ${ }^{57}$, T. Nanut ${ }^{49}$, I. Nasteva ${ }^{2}$, M. Needham ${ }^{58}$, I. Neri $^{21, e}$, N. Neri ${ }^{25, h}$, S. Neubert ${ }^{75}$, N. Neufeld ${ }^{48}$, R. Newcombe ${ }^{61}$, T. D. Nguyen ${ }^{49}$, C. Nguyen-Mau ${ }^{49, w}$, E. M. Niel ${ }^{11}$, S. Nieswand ${ }^{14}$, N. Nikitin ${ }^{40}$, N. S. Nolte ${ }^{48}$, C. Nunez ${ }^{85}$, A. Oblakowska-Mucha ${ }^{34}$, V. Obraztsov ${ }^{44}$, D. P. O'Hanlon ${ }^{54}$, R. Oldeman², ${ }^{27}$, M. E. Olivares ${ }^{68}$, C. J. G. Onderwater ${ }^{78}$, A. Ossowska ${ }^{35}$, J. M. Otalora Goicochea ${ }^{2}$, T. Ovsiannikova ${ }^{41}$, P. Owen ${ }^{50}$, A. Oyanguren ${ }^{47}$, B. Pagare ${ }^{56}$, P. R. Pais ${ }^{48}$, T. Pajero ${ }^{29,1,48}$, A. Palano ${ }^{19}, \quad$ M. Palutan ${ }^{23}, \quad$ Y. Pan ${ }^{62}, \quad$ G. Panshin ${ }^{83}$, A. Papanestis ${ }^{57}$, M. Pappagallo ${ }^{19, b}$, L. L. Pappalardo ${ }^{21, e}$, C. Pappenheimer ${ }^{65}$, W. Parker ${ }^{66}$, C. Parkes ${ }^{62}$, C. J. Parkinson ${ }^{46}$, B. Passalacqua ${ }^{21}$, G. Passaleva ${ }^{22}$, A. Pastore ${ }^{19}$, M. Patel ${ }^{61}$, C. Patrignani ${ }^{20, c}$, C. J. Pawley ${ }^{79}$, A. Pearce ${ }^{48}$, A. Pellegrino ${ }^{32}$, M. Pepe Altarelli ${ }^{48}$, S. Perazzini ${ }^{20}$, D. Pereima ${ }^{41}$, P. Perret ${ }^{9}$, K. Petridis ${ }^{54}$, A. Petrolini ${ }^{24, g}$, A. Petrov ${ }^{80}$, S. Petrucci ${ }^{58}$, M. Petruzzo ${ }^{25}$, A. Philippov ${ }^{42}$, L. Pica $^{29}$, M. Piccini ${ }^{77}$, B. Pietrzyk ${ }^{8}$, G. Pietrzyk ${ }^{49}$, M. Pili ${ }^{63}$, D. Pinci ${ }^{30}$, F. Pisani ${ }^{48}$, A. Piucci ${ }^{17}$, Resmi P.K ${ }^{10}$, V. Placinta ${ }^{37}$, J. Plews ${ }^{53}$, M. Plo Casasus ${ }^{46}$, F. Polci ${ }^{13}$, M. Poli Lener ${ }^{23}$, M. Poliakova ${ }^{68}$, A. Poluektov ${ }^{10}$, N. Polukhina ${ }^{82, t}$, I. Polyakov ${ }^{68}$, E. Polycarpo ${ }^{2}$, G. J. Pomery ${ }^{54}$, S. Ponce ${ }^{48}$, D. Popov ${ }^{6,48}$, S. Popov ${ }^{42}$, S. Poslavskii ${ }^{44}$, K. Prasanth ${ }^{35}$, L. Promberger ${ }^{48}$, C. Prouve ${ }^{46}$, V. Pugatch ${ }^{52}$, H. Pullen ${ }^{63}$, G. Punzi ${ }^{29, m}$, W. Qian ${ }^{6}$, J. Qin ${ }^{6}$, R. Quagliani ${ }^{13}$ ， B. Quintana ${ }^{8}$ ， N. V. Raab ${ }^{18}$, R. I. Rabadan Trejo ${ }^{10}$, B. Rachwal ${ }^{34}$, J. H. Rademacker ${ }^{54}$, M. Rama ${ }^{29}$, M. Ramos Pernas ${ }^{56}$, M. S. Rangel ${ }^{2}$, F. Ratnikov ${ }^{42,81}$, G. Raven ${ }^{33}$, M. Reboud ${ }^{8}$, F. Redi' ${ }^{49}$, F. Reiss ${ }^{13}$, C. Remon Alepuz ${ }^{47}$, Z. Ren ${ }^{3}$, V. Renaudin ${ }^{63}$, R. Ribatti ${ }^{29}$, S. Ricciardi ${ }^{57}$, D. S. Richards ${ }^{57}$, K. Rinnert ${ }^{60}$, P. Robbe ${ }^{11}$, A. Robert ${ }^{13}$, G. Robertson ${ }^{58}$, A. B. Rodrigues ${ }^{49}$, E. Rodrigues ${ }^{60}$, J. A. Rodriguez Lopez ${ }^{74}$, A. Rollings ${ }^{63}$, P. Roloff ${ }^{48}$, V. Romanovskiy ${ }^{44}, \quad$ M. Romero Lamas ${ }^{46}$, A. Romero Vidal ${ }^{46}$, J. D. Roth ${ }^{85}, \quad$ M. Rotondo ${ }^{23}$, M. S. Rudolph ${ }^{68}$, T. Ruf ${ }^{48}$ ，J. Ruiz Vidal ${ }^{47}$ ，A. Ryzhikov ${ }^{81}$ ，J. Ryzka ${ }^{34}$ ，J. J. Saborido Silva ${ }^{46}$ ，N. Sagidova ${ }^{38 ， N . ~ S a h o o ~}{ }^{56}$ ，B. Saitta ${ }^{27, d}$, D. Sanchez Gonzalo ${ }^{45}$, C. Sanchez Gras ${ }^{32}$, R. Santacesaria ${ }^{30}$, C. Santamarina Rios ${ }^{46}$, M. Santimaria ${ }^{23}$, E. Santovetti ${ }^{31,0}$, D. $\operatorname{Saranin}^{82}$, G. Sarpis ${ }^{59}$, M. Sarpis ${ }^{75}$, A. Sarti ${ }^{30}$, C. Satriano ${ }^{30, n}$, A. Satta ${ }^{31}$, M. Saur ${ }^{15}$, D. Savrina ${ }^{40,41}$, H. Sazak ${ }^{9}$, L. G. Scantlebury Smead ${ }^{63}$, S. Schael ${ }^{14}$, M. Schellenberg ${ }^{15}$, M. Schiller ${ }^{59}$, H. Schindler ${ }^{48}$, M. Schmelling ${ }^{16}$, B. Schmidt ${ }^{48}$, O. Schneider ${ }^{49}$, A. Schopper ${ }^{48}$, M. Schubiger ${ }^{32}$, S. Schulte ${ }^{49}$, M. H. Schune ${ }^{11}$, R. Schwemmer ${ }^{48}$, B. Sciascia ${ }^{23}$, A. Sciubba ${ }^{23}$, S. Sellam ${ }^{46}$, A. Semennikov ${ }^{41}$, M. Senghi Soares ${ }^{33}$, A. Sergi ${ }^{48,53}$, N. Serra ${ }^{50}$, L. Sestini ${ }^{28}$, A. Seuthe ${ }^{15}$, P. Seyfert ${ }^{48}$, D. M. Shangase ${ }^{85}$, M. Shapkin ${ }^{44}$, I. Shchemerov ${ }^{82}$, L. Shchutska ${ }^{49}$, T. Shears ${ }^{60}$, L. Shekhtman ${ }^{43, u}$, Z. Shen ${ }^{5}$, V. Shevchenko ${ }^{80}$, E. B. Shields ${ }^{26, i}$, E. Shmanin ${ }^{82}$, J. D. Shupperd ${ }^{68}$, B. G. Siddi ${ }^{21}$, R. Silva Coutinho ${ }^{50}$, G. Simi ${ }^{28}$, S. Simone ${ }^{19, b}$ ， I. Skiba ${ }^{21, e}$ ， N. Skidmore ${ }^{62}$, T. Skwarnicki ${ }^{68}$, M. W. Slater ${ }^{53}$, J. C. Smallwood ${ }^{63}$, J. G. Smeaton ${ }^{55}$, 
A. Smetkina ${ }^{41}$, E. Smith ${ }^{14}$, M. Smith ${ }^{61}$, A. Snoch ${ }^{32}$, M. Soares ${ }^{20}$, L. Soares Lavra ${ }^{9}$, M. D. Sokoloff ${ }^{65}$, F. J. P. Soler ${ }^{59}$, A. Solovev ${ }^{38}$, I. Solovyev ${ }^{38}$, F. L. Souza De Almeida ${ }^{2}$, B. Souza De Paula ${ }^{2}$, B. Spaan ${ }^{15}$, E. Spadaro Norella ${ }^{25, h}$, P. Spradlin ${ }^{59}$ ，F. Stagni ${ }^{48}$, M. Stahl ${ }^{65}$ ， S. Stahl ${ }^{48}$, P. Stefko ${ }^{49}$ ，O. Steinkamp ${ }^{50,82}$, S. Stemmle ${ }^{17}$, O. Stenyakin ${ }^{44}$, H. Stevens ${ }^{15}$, S. Stone ${ }^{68}$, M. E. Stramaglia ${ }^{49}$, M. Straticiuc ${ }^{37}$, D. Strekalina ${ }^{82}$, S. Strokov ${ }^{83}$, F. Suljik ${ }^{63}$, J. Sun ${ }^{27}$, L. Sun ${ }^{73}$, Y. Sun ${ }^{66}$, P. Svihra ${ }^{62}$, P. N. Swallow ${ }^{53}$, K. Swientek ${ }^{34}$, A. Szabelski ${ }^{36}$, T. Szumlak ${ }^{34}$, M. Szymanski ${ }^{48}$, S. Taneja ${ }^{62}$, F. Teubert ${ }^{48}$, E. Thomas ${ }^{48}$, K. A. Thomson ${ }^{60}$, M. J. Tilley ${ }^{61}$, V. Tisserand ${ }^{9}$, S. T'Jampens ${ }^{8}$, M. Tobin ${ }^{4}$, S. Tolk ${ }^{48}$, L. Tomassetti ${ }^{21, e}$, D. Torres Machado ${ }^{1}$, D. Y. Tou ${ }^{13}$, M. Traill ${ }^{59}$, M. T. Tran ${ }^{49}$, E. Trifonova ${ }^{82}$, C. Trippl ${ }^{49}$, G. Tuci ${ }^{29, m}$, A. Tully ${ }^{49}$, N. Tuning ${ }^{32}$, A. Ukleja ${ }^{36}$, D. J. Unverzagt ${ }^{17}$, A. Usachov ${ }^{32}$, A. Ustyuzhanin ${ }^{42,81}$, U. Uwer ${ }^{17}$, A. Vagner ${ }^{83}$, V. Vagnoni ${ }^{20}$, A. Valassi ${ }^{48}$, G. Valenti ${ }^{20}$, N. Valls Canudas ${ }^{45}$, M. van Beuzekom ${ }^{32}$, E. van Herwijnen ${ }^{82}$, C. B. Van Hulse ${ }^{18}$, M. van Veghel ${ }^{78}$, R. Vazquez Gomez ${ }^{46}$, P. Vazquez Regueiro ${ }^{46}$, C. Vázquez Sierra ${ }^{48}$, S. Vecchi ${ }^{21}$, J. J. Velthuis ${ }^{54}$, M. Veltri ${ }^{22, q}$, A. Venkateswaran ${ }^{68}$, M. Veronesi ${ }^{32}$, M. Vesterinen ${ }^{56}$, D. Vieira ${ }^{65}$, M. Vieites Diaz ${ }^{49}$, H. Viemann ${ }^{76}$, X. Vilasis-Cardona ${ }^{84}$, E. Vilella Figueras ${ }^{60}$, P. Vincent ${ }^{13}$, G. Vitali ${ }^{29}$, A. Vollhardt ${ }^{50}$, D. Vom Bruch ${ }^{13}$, A. Vorobyev ${ }^{38}$, V. Vorobyev ${ }^{43, u}$, N. Voropaev ${ }^{38}$, R. Waldi ${ }^{76}$, J. Walsh ${ }^{29}$, C. Wang ${ }^{17}$, J. Wang ${ }^{73}$, J. Wang ${ }^{3}$, J. Wang ${ }^{5}$, J. Wang ${ }^{4}$, M. Wang ${ }^{3}$, R. Wang ${ }^{54}$, Y. Wang ${ }^{7}$, Z. Wang ${ }^{50}$, H. M. Wark ${ }^{60}$, N. K. Watson ${ }^{53}$, S. G. Weber ${ }^{13}$, D. Websdale ${ }^{61}$, C. Weisser ${ }^{64}$, B. D. C. Westhenry ${ }^{54}$, D. J. White ${ }^{62}$, M. Whitehead ${ }^{54}$, D. Wiedner ${ }^{15}$, G. Wilkinson ${ }^{63}$, M. Wilkinson ${ }^{68}$, I. Williams ${ }^{55}$, M. Williams ${ }^{64,69}$, M. R. J. Williams ${ }^{58}$, F. F. Wilson ${ }^{57}$, W. Wislicki ${ }^{36}$, M. Witek ${ }^{35}$, L. Witola ${ }^{17}$, G. Wormser ${ }^{11}$, S. A. Wotton ${ }^{55}$, H. Wu ${ }^{68}$, K. Wyllie ${ }^{48}$, Z. Xiang ${ }^{6}$, D. Xiao ${ }^{7}$, Y. Xie ${ }^{7}$, A. $\mathrm{Xu}^{5}$, J. Xu ${ }^{6}$, L. $\mathrm{Xu}^{3}$, M. Xu ${ }^{7}$, Q. Xu ${ }^{6}$, Z. Xu ${ }^{6}$,

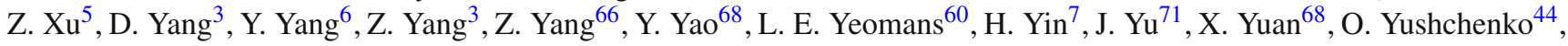
K. A. Zarebski ${ }^{53}$, M. Zavertyaev ${ }^{16, t}$, M. Zdybal ${ }^{35}$, O. Zenaiev ${ }^{48}$, M. Zeng ${ }^{3}$, D. Zhang ${ }^{7}$, L. Zhang ${ }^{3}$, S. Zhang ${ }^{5}$, Y. Zhang ${ }^{5}$, Y. Zhang ${ }^{63}$, A. Zhelezov ${ }^{17}$, Y. Zheng ${ }^{6}$, X. Zhou ${ }^{6}$, Y. Zhou ${ }^{6}$, X. Zhu ${ }^{3}$, V. Zhukov ${ }^{14,40}$, J. B. Zonneveld ${ }^{58}$, S. Zucchelli ${ }^{20, c}$, D. Zuliani ${ }^{28}$, G. Zunica ${ }^{62}$

${ }^{1}$ Centro Brasileiro de Pesquisas Físicas (CBPF), Rio de Janeiro, Brazil

${ }^{2}$ Universidade Federal do Rio de Janeiro (UFRJ), Rio de Janeiro, Brazil

${ }^{3}$ Center for High Energy Physics, Tsinghua University, Beijing, China

${ }^{4}$ Institute Of High Energy Physics (IHEP), Beijing, China

${ }^{5}$ School of Physics State Key Laboratory of Nuclear Physics and Technology, Peking University, Beijing, China

${ }^{6}$ University of Chinese Academy of Sciences, Beijing, China

${ }^{7}$ Institute of Particle Physics, Central China Normal University, Wuhan, Hubei, China

${ }^{8}$ Univ. Grenoble Alpes, Univ. Savoie Mont Blanc, CNRS, IN2P3-LAPP, Annecy, France

${ }^{9}$ Université Clermont Auvergne, CNRS/IN2P3, LPC, Clermont-Ferrand, France

${ }^{10}$ Aix Marseille Univ, CNRS/IN2P3, CPPM, Marseille, France

${ }^{11}$ Université Paris-Saclay, CNRS/IN2P3, IJCLab, Orsay, France

${ }^{12}$ Laboratoire Leprince-ringuet (llr), Palaiseau, France

${ }^{13}$ LPNHE, Sorbonne Université, Paris Diderot Sorbonne Paris Cité, CNRS/IN2P3, Paris, France

${ }^{14}$ I. Physikalisches Institut, RWTH Aachen University, Aachen, Germany

${ }^{15}$ Fakultät Physik, Technische Universität Dortmund, Dortmund, Germany

${ }^{16}$ Max-Planck-Institut für Kernphysik (MPIK), Heidelberg, Germany

${ }^{17}$ Physikalisches Institut, Ruprecht-Karls-Universität Heidelberg, Heidelberg, Germany

${ }^{18}$ School of Physics, University College Dublin, Dublin, Ireland

${ }^{19}$ INFN Sezione di Bari, Bari, Italy

${ }^{20}$ INFN Sezione di Bologna, Bologna, Italy

${ }^{21}$ INFN Sezione di Ferrara, Ferrara, Italy

22 INFN Sezione di Firenze, Firenze, Italy

${ }^{23}$ INFN Laboratori Nazionali di Frascati, Frascati, Italy

${ }^{24}$ INFN Sezione di Genova, Genova, Italy

${ }^{25}$ INFN Sezione di Milano, Milano, Italy

${ }^{26}$ INFN Sezione di Milano-Bicocca, Milano, Italy

${ }^{27}$ INFN Sezione di Cagliari, Monserrato, Italy

${ }^{28}$ Universita degli Studi di Padova, Universita e INFN, Padova, Padova, Italy

${ }^{29}$ INFN Sezione di Pisa, Pisa, Italy

${ }^{30}$ INFN Sezione di Roma La Sapienza, Roma, Italy

${ }^{31}$ INFN Sezione di Roma Tor Vergata, Roma, Italy 
32 Nikhef National Institute for Subatomic Physics, Amsterdam, Netherlands

${ }^{33}$ Nikhef National Institute for Subatomic Physics and VU University Amsterdam, Amsterdam, Netherlands

${ }^{34}$ AGH - University of Science and Technology, Faculty of Physics and Applied Computer Science, Kraków, Poland

35 Henryk Niewodniczanski Institute of Nuclear Physics Polish Academy of Sciences, Kraków, Poland

36 National Center for Nuclear Research (NCBJ), Warsaw, Poland

${ }^{37}$ Horia Hulubei National Institute of Physics and Nuclear Engineering, Bucharest-Magurele, Romania

38 Petersburg Nuclear Physics Institute NRC Kurchatov Institute (PNPI NRC KI), Gatchina, Russia

${ }^{39}$ Institute for Nuclear Research of the Russian Academy of Sciences (INR RAS), Moscow, Russia

${ }^{40}$ Institute of Nuclear Physics, Moscow State University (SINP MSU), Moscow, Russia

${ }^{41}$ Institute of Theoretical and Experimental Physics NRC Kurchatov Institute (ITEP NRC KI), Moscow, Russia

42 Yandex School of Data Analysis, Moscow, Russia

43 Budker Institute of Nuclear Physics (SB RAS), Novosibirsk, Russia

${ }^{44}$ Institute for High Energy Physics NRC Kurchatov Institute (IHEP NRC KI), Protvino, Russia, Protvino, Russia

45 ICCUB, Universitat de Barcelona, Barcelona, Spain

${ }^{46}$ Instituto Galego de Física de Altas Enerxías (IGFAE), Universidade de Santiago de Compostela, Santiago de Compostela, Spain

${ }^{47}$ Instituto de Fisica Corpuscular, Centro Mixto Universidad de Valencia - CSIC, Valencia, Spain

${ }^{48}$ European Organization for Nuclear Research (CERN), Geneva, Switzerland

${ }^{49}$ Institute of Physics, Ecole Polytechnique Fédérale de Lausanne (EPFL), Lausanne, Switzerland

50 Physik-Institut, Universität Zürich, Zürich, Switzerland

51 NSC Kharkiv Institute of Physics and Technology (NSC KIPT), Kharkiv, Ukraine

${ }^{52}$ Institute for Nuclear Research of the National Academy of Sciences (KINR), Kyiv, Ukraine

53 University of Birmingham, Birmingham, UK

${ }^{54}$ H.H. Wills Physics Laboratory, University of Bristol, Bristol, UK

55 Cavendish Laboratory, University of Cambridge, Cambridge, UK

56 Department of Physics, University of Warwick, Coventry, UK

57 STFC Rutherford Appleton Laboratory, Didcot, UK

58 School of Physics and Astronomy, University of Edinburgh, Edinburgh, UK

59 School of Physics and Astronomy, University of Glasgow, Glasgow, UK

${ }^{60}$ Oliver Lodge Laboratory, University of Liverpool, Liverpool, UK

${ }^{61}$ Imperial College London, London, UK

62 Department of Physics and Astronomy, University of Manchester, Manchester, UK

63 Department of Physics, University of Oxford, Oxford, UK

${ }^{64}$ Massachusetts Institute of Technology, Cambridge, MA, USA

${ }^{65}$ University of Cincinnati, Cincinnati, OH, USA

66 University of Maryland, College Park, MD, USA

${ }^{67}$ Los Alamos National Laboratory (LANL), Los Alamos, USA

68 Syracuse University, Syracuse, NY, USA

${ }^{69}$ School of Physics and Astronomy, Monash University, Melbourne, Australia

${ }^{70}$ Pontifícia Universidade Católica do Rio de Janeiro (PUC-Rio), Rio de Janeiro, Brazil

71 Physics and Micro Electronic College, Hunan University, Changsha City, China

72 Guangdong Provencial Key Laboratory of Nuclear Science, Institute of Quantum Matter, South China Normal University, Guangzhou, China

73 School of Physics and Technology, Wuhan University, Wuhan, China

${ }^{74}$ Departamento de Fisica, Universidad Nacional de Colombia, Bogota, Colombia

75 Universität Bonn-Helmholtz-Institut für Strahlen und Kernphysik, Bonn, Germany

${ }^{76}$ Institut für Physik, Universität Rostock, Rostock, Germany

77 INFN Sezione di Perugia, Perugia, Italy

78 Van Swinderen Institute, University of Groningen, Groningen, Netherlands

${ }^{79}$ Universiteit Maastricht, Maastricht, Netherlands

${ }^{80}$ National Research Centre Kurchatov Institute, Moscow, Russia

${ }^{81}$ National Research University Higher School of Economics, Moscow, Russia

${ }^{82}$ National University of Science and Technology "MISIS”, Moscow, Russia 
${ }^{83}$ National Research Tomsk Polytechnic University, Tomsk, Russia

${ }^{84}$ DS4DS, La Salle, Universitat Ramon Llull, Barcelona, Spain

${ }^{85}$ University of Michigan, Ann Arbor, USA

${ }^{a}$ Universidade Federal do Triângulo Mineiro (UFTM), Uberaba-MG, Brazil

${ }^{\mathrm{b}}$ Università di Bari, Bari, Italy

${ }^{\mathrm{c}}$ Università di Bologna, Bologna, Italy

${ }^{\mathrm{d}}$ Università di Cagliari, Cagliari, Italy

${ }^{\mathrm{e}}$ Università di Ferrara, Ferrara, Italy

${ }^{\mathrm{f}}$ Università di Firenze, Firenze, Italy

${ }^{\mathrm{g}}$ Università di Genova, Genova, Italy

${ }^{\mathrm{h}}$ Università degli Studi di Milano, Milano, Italy

${ }^{\mathrm{i}}$ Università di Milano Bicocca, Milano, Italy

j Università di Modena e Reggio Emilia, Modena, Italy

${ }^{\mathrm{k}}$ Università di Padova, Padova, Italy

${ }^{1}$ Scuola Normale Superiore, Pisa, Italy

${ }^{\mathrm{m}}$ Università di Pisa, Pisa, Italy

${ }^{\mathrm{n}}$ Università della Basilicata, Potenza, Italy

${ }^{\circ}$ Università di Roma Tor Vergata, Roma, Italy

$\mathrm{p}$ Università di Siena, Siena, Italy

q Università di Urbino, Urbino, Italy

${ }^{\mathrm{r}} \mathrm{MSU}$ - Iligan Institute of Technology (MSU-IIT), Iligan, Philippines

${ }^{s}$ AGH - University of Science and Technology, Faculty of Computer Science, Electronics and Telecommunications, Kraków, Poland

${ }^{t}$ P.N. Lebedev Physical Institute, Russian Academy of Science (LPI RAS), Moscow, Russia

" Novosibirsk State University, Novosibirsk, Russia

${ }^{\mathrm{v}}$ Department of Physics and Astronomy, Uppsala University, Uppsala, Sweden

${ }^{\mathrm{w}}$ Hanoi University of Science, Hanoi, Vietnam 